\title{
STABILITY OF GASEOUS STARS IN SPHERICALLY SYMMETRIC MOTIONS*
}

\author{
SONG-SUN LIN ${ }^{\dagger}$
}

\begin{abstract}
We study the linearized stability of stationary solutions of gaseous stars which are in spherically symmetric and isentropic motion. If viscosity is ignored, we have following three types of problems: (EC), Euler equation with a solid core; (EP), Euler-Poisson equation without a solid core; (EPC), Euler-Poisson equation with a solid core. In Lagrangian formulation, we prove that any solution of (EC) is neutrally stable. Any solution of (EP) and (EPC) is also neutrally stable when the adiabatic index $\gamma \in\left(\frac{4}{3}, 2\right)$ and unstable for (EP) when $\gamma \in\left(1, \frac{4}{3}\right)$. Moreover, for (EPC) and $\gamma \in(1,2)$, any solution with small total mass is also neutrally stable. When viscosity is present $(\nu>0)$, the velocity disturbance on the outer surface of gas is important. For $\nu>0$, we prove that the neutrally stable solution (when $\nu=0$ ) is now stable with respect to positive-type disturbances, which include Dirichlet and Neumann boundary conditions. The solution can be unstable with respect to disturbances of some other types. The problems were studied through spectral analysis of the linearized operators with singularities at the endpoints of intervals.
\end{abstract}

Key words. stability, isentropic gas, self-gravitating, solid core, limit-point singularity

AMS subject classifications. 35J65, 35P30, 85A15, 85A20

PII. S0036141095292883

1. Introduction. In this paper, we shall study the stability problem of gaseous stars which are in spherically symmetric and isentropic motion. The problem originated in Newtonian (nonrelativistic) astrophysical theory. A model equation for describing such motion is shown below:

$$
\begin{gathered}
\frac{\partial \rho}{\partial t}+v \frac{\partial \rho}{\partial r}+\rho \frac{\partial v}{\partial r}+\frac{2}{r} \rho v=0 \\
\rho\left(\frac{\partial v}{\partial t}+v \frac{\partial v}{\partial r}\right)+\frac{\partial p}{\partial r}=-\frac{\rho}{r^{2}}\left\{M_{0}+4 \pi \delta \int_{R_{0}}^{r} \rho(t, s) s^{2} d s\right\} \\
+\nu\left\{\begin{array}{l}
\partial^{2} v \\
\partial r^{2}
\end{array}+\frac{2 \partial v}{r} \partial r-\frac{2}{r^{2}} v\right\}, \\
p=A \rho^{\gamma},
\end{gathered}
$$

where $t \geq 0$ and $0 \leq R_{0}<r<\infty$; see, e.g., $[6,7,8,9,10,11,12,13,14,20]$. Here the unknown variable $\rho$ is the density of the gas and $v$ is the outward velocity. $p$ is the pressure, $A$ is a positive constant which is related to entropy, and $\gamma \in(1,2)$ is the adiabatic exponent.

The explanation of the physical parameters $\delta, M_{0}, R_{0}$, and $\nu$ is as follows:

$\delta$ is the effect of self-gravitating of gas, the mutual graviational attraction among gas molecules, and is assumed to be either 0 or 1 . If $\delta=0$, we ignore the effect of self-gravitating. This may happen when the total amount of gas is relatively small. If $\delta=1$, we then consider the self-gravitating of gas to be important.

* Received by the editors October 6, 1995; accepted for publication January 31, 1996 . This research was partially supported by the National Science Council of the Republic of China.

http://www.siam.org/journals/sima/28-3/29288.html

$\dagger$ Department of Applied Mathematics, National Chiao Tung University, Hsin-chu 30050, Taiwan, Republic of China (sslin@math.nctu.edu.tw). 
$M_{0}$ is the total mass of the solid core surrounded by the gas. If $M_{0}=0$, then we assume that $R_{0}=0$. This is the case when there is no solid core and also no vacuum in the central part of the gaseous body. If $M_{0}>0$, we assume that there is a stationary, spherical solid core surrounded by the gas. In this case, we normalize the radius of the solid core with $R_{0}=1$. We also assume that the gas is in contact the surface of the solid core, i.e., no vacuum exists between the core and the gas. A nonslip condition is now imposed at the interface, i.e.,

$$
v(t, 1)=0 \quad \text { for } t \geq 0 .
$$

We note that astrophysicists consider the solid core to be made of condensed gases in which there may be complicated activity that influences the surrounding gas. However, for mathematical simplicity, we will consider these condensed gases to be a solid core and ignore their influence on the surface gas.

$\nu$ is viscosity coefficient. We are mainly concered with inviscid flow, i.e., $\nu=0$. After presenting a detailed study of inviscid flow, we will discuss the effect of viscosity on the stability of stationary solutions.

If viscosity is ignored, then according to the different combinations of parameters $\delta, M_{0}$, and $R_{0}$, we have following three types of problems:

(EC): Euler equation with solid core $\left(\delta=0, M_{0}>0, R_{0}=1, \nu=0\right)$;

(EP): Euler-Poisson equation without solid core $\left(\delta=1, M_{0}=0, R_{0}=0, \nu=0\right)$;

(EPC): Euler-Poisson equation with solid core $\left(\delta=1, M_{0}>0, R_{0}=1, \nu=0\right)$.

If viscosity is present, i.e., $\nu>0$, then the Euler equation will be replaced by a NavierStokes equation and we have problems (NSC), (NSP), and (NSPC), respectively.

The stationary solution $(\rho(r), 0)$ of $(1.1)-(1.3)$ satisfies

$$
\frac{d p}{d r}=-\frac{\rho}{r^{2}}\left\{M_{0}+4 \pi \delta \int_{R_{0}}^{r} \rho(s) s^{2}\right\} .
$$

If we introduce the variable $u(r)$ and the parameter $\mu>0$ in

$$
\rho=C_{\gamma} u^{q} \quad \text { and } \quad \mu=d_{\gamma} M_{0},
$$

where

$$
q=\frac{1}{\gamma-1}, \quad C_{\gamma}=\left\{\begin{array}{c}
A \gamma \\
4 \pi(\gamma-1)
\end{array}\right\}^{2-\gamma}, \quad \text { and } \quad d_{\gamma}=\left\{(4 \pi)^{\gamma-1} \frac{\gamma-1}{A \gamma}\right\}^{2-\gamma}
$$

then (1.5) and (1.4) can be studied by considering the following initial-value problems: for (EC),

$$
\left.\begin{array}{l}
u^{\prime \prime}+\frac{2}{r} u^{\prime}=0, \quad r>1, \\
u(1, \alpha, \mu)=\alpha \quad \text { and } \quad u^{\prime}(1, \alpha, \mu)=-\mu
\end{array}\right\}
$$

for (EP),

$$
\left.\begin{array}{l}
u^{\prime \prime}+\frac{2}{r} u^{\prime}+u^{q}=0, \quad r>0, \\
u(0, \alpha)=\alpha \quad \text { and } \quad u^{\prime}(0, \alpha)=0
\end{array}\right\}
$$

and for $(\mathrm{EPC})$,

$$
\left.\begin{array}{l}
u^{\prime \prime}+\frac{2}{r} u^{\prime}+u^{q}=0, \quad r>1, \\
u(1, \alpha, \mu)=\alpha \quad \text { and } \quad u^{\prime}(1, \alpha, \mu)=-\mu
\end{array}\right\} .
$$


Here $\alpha>0$ is taken as a shooting parameter.

The total mass of the stationary solution $u$ is given by

$$
\tilde{M}(u)=4 \pi C_{\gamma} \int_{R_{0}}^{R} u^{q}(r) r^{2} d r
$$

where $R \in\left(R_{0}, \infty\right]$ is the first zero of $u$, i.e.,

$$
u(R)=0 \quad \text { and } \quad u(r)>0 \quad \text { in }\left(R_{0}, R\right) .
$$

From a physical point of view, we are only interested in a stationary solution with finite total mass.

The solution of (1.6) with finite total mass can be written explicitly as

$$
u=\mu\left(\frac{1}{r}-\frac{1}{R}\right)
$$

for some $R \in(1, \infty]$.

The solution of (1.7) has been studied extensively by Lane et al.; see, e.g., [1]. Their solutions include the ball type $(R<\infty)$, the ground-state type $(R=+\infty)$, and the singularity type, i.e., $\lim _{r \rightarrow 0^{+}} u(r)=\infty$.

Equation (1.8) has recently been studied in [5] and may have multiple solutions for certain $\mu$ and $\tilde{M}$ when $q>3$.

The multiplicity results of these problems will be given in section 2 .

In this paper, we mainly study the stability of stationary solutions obtained from (1.6), (1.7), and (1.8) since only the local existence and not the global-existance of the initial-value problem in (1.1)-(1.3) is known (see, e.g., [6, 7, 8, 9, 10,11,12,13,14]). We therefore need only study the linearized stability of these stationary solutions.

The linearized stability problem of the stationary solution $\rho(r)$ will be studied in Lagrangian formulation. Indeed, equations (1.1)-(1.3) can be written in Lagrangian coordinates as

$$
\begin{gathered}
\rho_{t}+4 \pi \rho\left(r^{2} v\right)_{x}=0 \\
v_{t}+4 \pi r^{2} p_{x}+\frac{1}{r^{2}}\left(M_{0}+x\right)=16 \pi^{2} \nu\left(r^{2} \rho v_{x}\right)_{x}-2 \nu v\left(r^{2} \rho\right)^{-1}, \\
r=\left\{R_{0}+\frac{3}{4 \pi} \int_{0}^{x} \frac{1}{\rho(t, y)} d y\right\}^{\frac{1}{3}} \text { and } x=4 \pi \int_{R_{0}}^{r} \rho(s, t) s^{2} d s
\end{gathered}
$$

where $t \geq 0$ and $x \in(0, \tilde{M})$. We assume that the perturbation of $(\rho(x), 0)$ is in a radial direction only and write

$$
\rho(t, x)=\rho(x)\left\{1+\varepsilon e^{\lambda t} \Phi(x)\right\} \quad \text { and } \quad v(t, x)=\varepsilon e^{\lambda t} \Psi(x)
$$

in (1.11) and (1.12), where $|\varepsilon|$ is small. Let

$$
\phi(x)=\int_{0}^{x} \frac{\Phi(y)}{\rho(y)} d y .
$$


Then the linear equations for $\Phi$ and $\Psi$ can be simplified as follows:

$$
\begin{aligned}
\left(\rho p \phi_{x}\right)_{x}-\frac{1}{\pi \gamma r^{3}} p_{x} \phi= & \frac{\lambda^{2}}{\gamma\left(4 \pi r^{2}\right)^{2}} \phi \\
& -\frac{\lambda \nu}{4 \pi \gamma r^{2}}\left\{16 \pi^{2}\left[r^{4} \rho\left(\frac{1}{4 \pi r^{2}} \phi\right)_{x}\right]_{x}-\frac{2}{r^{2} \rho} \frac{1}{4 \pi r^{2}} \phi\right\}
\end{aligned}
$$

with boundary condition

$$
\phi(0)=0,
$$

where

$$
r=\left\{1+\frac{3}{4 \pi} \int_{0}^{x} \frac{1}{\rho(y)} d y\right\} .
$$

Transforming (1.14) into $r$-coordinates and writting $\phi(x)=\psi(r)$, we obtain

$$
L \psi \equiv\left(r^{-2} p \psi^{\prime}\right)^{\prime}-\frac{4}{\gamma} r^{-3} p^{\prime} \psi=\frac{\lambda^{2}}{\gamma} r^{-2} \rho \psi-\frac{\lambda \nu}{\gamma}\left(r^{-2} \psi^{\prime}\right)^{\prime}
$$

with $\psi\left(R_{0}\right)=0$, where $p$ is the pressure in $r$-coordinates. Since $\rho(R)=0$, if $\nu=0$, then (1.16) is singular at $r=R$. We can prove that the singularity at $R$ is a limitpoint type and so $L$ is self-adjoint. Therefore, $\lambda^{2}$ is real for any eigenvalue $\lambda$ when $\nu=0$. Now $\rho$ is called neutrally stable if $\lambda^{2}<0$ for any eigenvalue $\lambda$ and unstable if $\lambda_{1}^{2}>0$ for some eigenvalue $\lambda_{1}$. Hence if $\nu=0$, then neutrally stable is the best we can hope for. Indeed, when $\nu=0$, we have our stability results for ball-type solutions as follows.

THEOREM 1.1. Assume that $\nu=0$ and ball-type solutions have been considered. Then

(I) any solution of (EC) is neutrally stable; and

(II) any solution of (EP) is neutrally stable if $q \in(1,3)$ and unstable if $q>3$;

(III) for (EPC), we have the following:

(i) any solution is neutrally stable if $q \in(1,3]$,

(ii) for any $q>1, u(\cdot, \alpha, \mu)$ is neutrally stable if $\alpha \in(0, \mu]$, and

(iii) if $|R-1|$ is sufficiently small, then it is neutrally stable.

Some stability results concerning ground-state-type and singularity-type solutions are also presented in section 4 .

When viscosity is present and $\lambda \notin\left[-{ }_{\nu}^{\gamma} p\left(R_{0}\right), 0\right]$, then (1.16) is regular at $R$. In this case, the viscosity term plays the dominant role in studying the eigenvalue problems. Now $\rho$ is called stable if $\operatorname{Re} \lambda<0$ for any eigenvalue $\lambda$ and unstable if $\operatorname{Re} \lambda_{1}>0$ for some eigenvalue $\lambda_{1}$. Note that (1.16) is genuinely quadratic in $\lambda$ (linear in $\lambda^{2}$ when $\nu=0$ ) and $\lambda$ is complex in general. Hence when $\nu>0$, we may have better than the neutral stability that we have when $\nu=0$. Since the outer surface of gas is a free surface, the velocity disturbance $\Psi$ on it will play an important role. For example, we have stability results for (EC), (EP), and (EPC) as follows.

THEOREM 1.2. Let u be a neutrally stable, ball-type stationary solution of (EC), $(\mathrm{EP})$, or $(\mathrm{EPC})$ when $\nu=0$. Then for any $\nu>0, u$ is stable with respect to $\Psi=$ $\psi_{1}+i \psi_{2}$ if $\psi_{j}(\tilde{M}) \psi_{j}^{\prime}(\tilde{M}) \leq 0$ for both $j=1$ and 2 on the gas surface. On the other hand, there is a positive constant $\kappa_{*}$ depending on $u$ such that $u$ is unstable with respect to $\Psi=\psi_{1}+i \psi_{2}$ if $\psi_{1}^{\prime}(\tilde{M}) / \psi_{1}(\tilde{M}) \geq \kappa_{*}$ and some $\psi_{2}$. 
The precise definition of stability with respect to the boundary disturbance $\Psi$ is given in section 5 .

The paper is organized as follows. In section 2 , we recall some useful multiplicity results for stationary solutions with finite total masses. Their stabilities will be investigated in subsequent sections. In section 3, we study the linearized operators $L$ and prove that they have limit-point-type singularities at their endpoints. We also provide a useful comparison lemma to test for stability. In section 4, we prove various stability results, which include Theorem 1.1. The solutions for other types of stability problems are also studied. In section 5 , we study the effect of viscosity on stability problems and prove some results, including Theorem 1.2. In Appendix A, we study the asymptotic behavior of solutions of (1.16) at $R$ when $\nu=0$, which is very useful for studying ball-type solutions. In Appendix B, we recall Friedrichs' criteria for the spectrum discreteness of differential operators that have singular endpoints. These criteria are very useful in studying ground-state-type and singularity-type solutions.

2. Stationary solutions. In this section, we recall some multiplicity results for stationary solutions without interior vacuums and with finite total masses. Let $R \leq \infty$ be the first zero of solution $u$ and $\tilde{M}(u)$ be the total mass given in (1.9). For notational simplicity, we omit the constant $4 \pi C_{\gamma}$ in (1.9) and then define

$$
M(u)=\int_{R_{0}}^{R} u(r)^{q} r^{2} d r,
$$

where $R_{0}=0$ for (EP) and $R_{0}=1$ for (EC) and (EPC).

Since the total mass of a gas remains constant while it is in motion and it may tend to a stationary state as time goes by, it is useful to know the numbers of stationary solutions for the same total mass. Hence we try to answer the following questions.

Questions. Given $M>0$, how many solutions $u$ are there for (EP) with $M(u)=$ $M$ ? Given $\mu>0$ and $M>0$, how many solutions $u$ are there for (EC) or (EPC) with $M(u)=M ?$

Complete answers of (EC) and (EP) can be provided; see, e.g., [1]. However, (EPC) has only recently been studied and the result is complete for $1<q \leq 3$ but partial when $q>3$; see [5].

First, for (EC), the solution of (1.6) is given by

$$
u(r, \alpha, \mu)=\alpha-\mu+\mu \frac{1}{r} .
$$

If $\alpha \in(0, \mu)$, then $u(R(\alpha, \mu), \alpha, \mu)=0$ with

$$
R(\alpha, \mu)=\left(1-\frac{\alpha}{\mu}\right)^{-1} .
$$

In this case, we may write $u(\cdot, \alpha, \mu)=u_{R, \mu}$ with

$$
u_{R, \mu}(r)=\mu\left(\frac{1}{r}-\frac{1}{R}\right) .
$$

It is clear that $M\left(u_{R, \mu}\right)$ is strictly increasing in $R$ and tends to

$$
M_{q}^{*}= \begin{cases}+\infty & \text { if } 1<q \leq 3, \\ \frac{1}{q-3} \cdot \mu^{q} & \text { if } q>3 .\end{cases}
$$


If $\alpha=\mu$, then $R(\mu, \mu)=+\infty$ and

$$
u(r, \mu, \mu)=\frac{\mu}{r}
$$

with

$$
M(u(\cdot, \mu, \mu))=M_{q}^{*} .
$$

If $\alpha>\mu$, then $M(u(\cdot, \alpha, \mu))=\infty$, which is not of physical interest. Hence we have the following unique result for (EC).

Proposition 2.1. For any $q>1, \mu>0$, and $M \in\left(0, M_{q}^{*}\right)$, there is a unique solution $u_{R, \mu}$ for $(\mathrm{EC})$ such that $M\left(u_{R, \mu}\right)=M$.

Next, for (EP), we consider the initial-value problem

$$
\begin{gathered}
u^{\prime \prime}+\frac{2}{r} u^{\prime}+u^{q}=0, \quad r>0, \\
u^{\prime}(0, \alpha)=0 \text { and } u(0, \alpha)=\alpha>0 .
\end{gathered}
$$

It is known that solutions of (2.3) have similar properties. Indeed, if $u(r)$ is a solution of (2.3), then for any $\beta>0$,

$$
u_{\beta}(r)=\beta^{\sigma} u(\beta r)
$$

is also a solution, where $\sigma=\frac{2}{q-1}$. The total mass of $u_{\beta}$ is

$$
M\left(u_{\beta}\right)=\beta^{3-q} M(u) .
$$

The property (2.5) is related to the following classical Lane-Emden-Fowler transformations:

Let

$$
r=e^{-\tau} \quad \text { and } \quad z(\tau)=r^{\sigma} u(r) .
$$

(2.3) can then be transformed into the autonomous equation

$$
z^{\prime \prime}+(2 \sigma-1) z^{\prime}+\sigma(\sigma-1) z+z^{q}=0
$$

or, equivalently, the dynamic system

$$
\left\{\begin{array}{l}
z^{\prime}=y \\
\left.y^{\prime}=-\{2 \sigma-1) y+\sigma(\sigma-1) z+z^{q}\right\} .
\end{array}\right.
$$

If $q \in(1,3]$, then $0=(0,0)$ is the only equilibrium for $(2.9)$ on the right half-plane $R_{+}^{2}=\{(z, y): z \geq 0\}$. If $q>3$, then there is another equilibrium $S=\left(z_{\sigma}, 0\right)$, where

$$
z_{\sigma}=\{\sigma(1-\sigma)\}^{2} .
$$

0 is always a saddle point with the unstable manifold $\Gamma$, which is leaving in the direction $(1,1-\sigma)^{t}$, and the stable manifold $\tilde{\Gamma}$, which is arriving for the direction $(1,-\sigma)$, where $(a, b)^{t}$ is the transpose of vector $(a, b)$ in $R^{2}$. Let

$$
q^{+}=1+\frac{2}{\sigma^{+}} \text {and } \quad \sigma^{+}=\sqrt{ } 2-\frac{1}{2} .
$$


It is than easy to verify that $q^{+} \in(3,5)$.

We now list some useful properties of the equilibrium $S$ and system (2.9) on the phase plane $R_{+}^{2}$.

PROPOSITION 2.2.

(I)

(i) If $q \in\left(3, q^{+}\right)$, then $S$ is a stable improper node.

(ii) If $q=q^{+}$, then $S$ is a stable proper node.

(iii) If $q \in\left(q^{+}, 5\right)$, then $S$ is stable spiral.

(iv) If $q=5$, then $S$ is a center.

(v) If $q>5$, then $S$ is an unstable spiral.

(i) For $q \in(3,5)$, the unstable manifold $\Gamma$ of 0 is a heteroclinic orbit connecting 0 and $S$. There is no nontrivial periodic orbit on $R_{+}^{2}$.

(ii) For $q=5, \Gamma=\tilde{\Gamma}$, i.e., $\Gamma$ is a homoclinic orbit of 0 . The inside of $\Gamma$ is covered by a family of concentric periodic orbits centered around 0 .

$S$.

(iii) For $q>5$, the stable manifold $\tilde{\Gamma}$ of 0 is a heteroclinic orbit connecting 0 and

The proofs are elementary and omitted; see [1] for details.

Every trajectory in the phase plane of (2.9) represents a family of self-similar solutions in (2.5). After carefully investigating the trajectories in the phase plane, we have exactly four types of solutions for (EP) with finite total mass for (EP):

(i) B-type solutions: ball-type solutions that lie on $\tilde{\Gamma}$ and appear when $q \in$

(ii) G-type solutions: ground-state solutions that also lie on $\tilde{\Gamma}$ and only appear when $q \geq 5$; they also have fast decay rates as $r \rightarrow+\infty$, i.e.,

$$
\lim _{r \rightarrow+\infty} r u(r) \in(0, \infty)
$$

(iii) SB-type solutions: ball-type solutions with a singularity at $r=0$ that appear when $q \in(3,5)$ and are trajectories between $\Gamma$ and $\tilde{\Gamma}$ that have a weak singularity, i.e., $u$ satisfies

$$
\lim _{r \rightarrow 0^{+}} r^{\sigma} u(r) \in(0, \infty)
$$

(iv) SG-type solutions: ground-state solutions with a singularity at $r=0$ that lie on $\Gamma$ and satisfy (2.12); they also appear when $q \in(3,5)$;

Note that if the singularity at $r=0$ is strong, i.e.,

$$
\lim _{r \rightarrow 0^{+}} r u(r)>0,
$$

then $u$ has an infinite total mass: for example, the SB-type solution when $q \in(1,3)$. If the ground-state solution has a slow-decay rate at $\infty$, i.e.,

$$
\lim _{r \rightarrow \infty} r^{\sigma} u(r)>0
$$

then $u$ also has an infinite total mass, which includes the following cases:

(i) $q_{\sigma} r^{-\sigma}$ for $q>3$; this corresponds to the equilibrium $S=\left(z_{\sigma}, 0\right)$;

(ii) when $q=5$, all trajectories lie on homoclinic orbit $\Gamma$;

(iii) when $q>5$, all trajectories spiral out from $S$; 
With this preparation complete, we can now state our unique results for (EP).

Proposition 2.3. For (EP), we have the following:

(i) If $q \in(1,3)$ and any $M>0$, there is a unique-B-type-solution $u$ such that $M(u)=M$.

(ii) If $q=3$, only a special $\hat{M}$ of a stationary solution $u-a$ B-type solutionadmits. (All similar solutions of $u$ also have the same total mass $\hat{M}$.)

(iii) If $q \in(3,5)$ and any $M>0$, there are unique B-type, SB-type, and SG-type solutions with the same total mass $M$.

(iv) If $q=5$ and any $M>0$, there is a unique-G-type-solution $u$ such that $M(u)=M$.

(v) If $q>5$, there is no stationary solution with finite total mass.

Proof. The proofs are based on the phase-plane analysis in (2.9) and the use of (2.6), and they are elementary. Thus the details are omitted.

As for (EPC), there are two types of solutions with finite total mass:

(i) BC-type solutions: ball-type solutions with solid cores;

(ii) GC-type solutions: ground-state solutions with solid cores that satisfy (2.11).

We recall some results from [5].

Proposition 2.4. For (EPC), we have the following:

(i) When $q \in(1,3]$, for any $\mu>0$ and $M>0$, there is a unique-BC-typesolution $u$ that satisfies $M(u)=M$.

(ii) When $q>3$, for any $\mu>0$, the solution set is the disjoint union of $N$ many connected components $C_{k}=\left\{u(\cdot, \alpha, \mu): \alpha \in\left(\tilde{\alpha}_{k}, \hat{\alpha}_{k}\right)\right\}, k=1,2, \ldots, N$, where $N=N(\mu, q)$ is a positive integer or infinity.

At $C_{k}$ with $k \geq 2, M((u(\cdot, \alpha, \mu))$ tends to infinity at at least one end. At $C_{1}, \tilde{\alpha}_{1}=0$ and $\hat{\alpha}_{1}>\mu$.

For detailed statements of Proposition 2.4(ii), see Theorems 3.5, 3.7, 3.9, and 3.13 in [5].

Remark 2.5. When there is a vaccum in the central part of the gaseous body that is also stationary, then $u$ satisfies

$$
\begin{aligned}
u^{\prime \prime}(r)+\frac{2}{r} u^{\prime}(r)+u^{q}(r) & =0, \quad R_{1}<r<R_{2}, \\
u\left(R_{1}\right)=0 & =u\left(R_{2}\right),
\end{aligned}
$$

where $0<R_{1}<R_{2} \leq \infty$. For any $q>1$ and $0<R_{1}<R_{2}<\infty$, Ni and Nussbaum [17] proved that there is a unique positive solution of (2.15) and (2.16). In contrast to Proposition 2.3(v), for any $q>1$, the solution $u$ of (2.15) and (2.16) with $R_{2}<\infty$ has a finite total mass. We can then ask the following questions: Given $q>1$ and $M>0$, how many solutions $u$ are there for (2.15) and (2.16) with $M(u)=M$ ? What is the stability of these annular-type solutions? These problems will be studied later.

3. Linearizations. In this section, we will use a Lagrangian formulation to study the stability of the stationary solutions obtained in last section. Since we want to know the stability result when the outer surface of the gas is also perturbed, it is convenient to work in Lagrangian coordinates. We study only the inviscid flow in this section and defer study of the the viscous flow to section 5 .

For notational simplicity, we replace $r$ with $r$ in (1.16) with $\nu=0$. We then obtain

$$
\mathcal{L} \psi=-\ell W \psi \quad \text { in }\left(R_{0}, R\right)
$$


where

$$
\mathcal{L} \psi \equiv\left(r^{-2} p \psi^{\prime}\right)^{\prime}-\frac{4}{\gamma} r^{-3} p^{\prime} \psi, \quad W(r) \equiv \frac{\rho(r)}{\gamma r^{2}}, \quad \text { and } \quad \ell=-\lambda^{2} .
$$

$\psi$ also satisfies the boundary condition

$$
\psi\left(R_{0}\right)=0 .
$$

In terms of $u,(3.1)$ can also be written as

$$
L_{0} \psi \equiv \psi^{\prime \prime}+\left\{(1+q) \frac{u^{\prime}}{u}-\frac{2}{r}\right\} \psi^{\prime}-\frac{4 q u^{\prime}}{r} \frac{u}{u}=-\ell\left(\gamma A C_{\gamma}^{\gamma-1}\right)^{-1} \frac{\psi}{u}
$$

Since $u(R)=0, \mathcal{L}$ is singular at $R$. Furthermore, $\mathcal{L}$ is also singular at $r=0$ for (EP).

When $R<\infty$, we first study the asymptotic behavior of solution $\psi$ of (3.1) at $R$. Indeed, we have the following result. (The proof is given in Appendix A.)

Lemma 3.1. Let $R<\infty$. If $\ell$ is real and $\psi$ is a (real) solution of $(3.1)$ in $\left(R_{0}, R\right)$, then either $\psi$ is bounded at $r=R$ or $\psi(r)=(R-r)^{-q} \hat{\psi}(r)$ for $r$ close to $R$, with $\hat{\psi}(R) \neq 0$, and $\hat{\psi}$ is continuous at $R$. Furthermore, in the former case, $\psi$ is $C^{2}$ at $R$, and in the latter case,

$$
\psi^{\prime}(r)=q(R-r)^{-q-1} \hat{\psi}(R)+o\left((R-r)^{-q-1}\right)
$$

as $r \rightarrow R^{-}$.

Similarly, if $R_{0}=0$, then either $\psi(0) \neq 0$ or $|\psi(r)| \leq C r^{3}$ for $r$ close to 0 and some $C>0$.

To study the singularity type at $R$, it is convenient to remove the weight function $W$ from right-hand side of (3.1). Indeed, if $R_{0}=1$, let $r_{0}=1$, and if $R_{0}=0$, choose any $r_{0} \in(0, R)$ and fix it. Then define

$$
s=s(r)=\int_{r_{0}}^{r} W(\tau) d \tau=\frac{1}{\gamma} \int_{r_{0}}^{r} \tau^{-2} \rho(\tau) d \tau
$$

and

$$
S_{0}=\int_{r_{0}}^{R_{0}} W(\tau) d \tau \quad \text { and } \quad S=\int_{r_{0}}^{R} W(\tau) d \tau
$$

It is clear that $S_{0}=0$ when $R_{0}=1$ and $S_{0}=-\infty$ when $R_{0}=0$. Furthermore, $W>0$ in $\left(R_{0}, R\right)$ implies that the inverse function of $s(r)$ exists. We may denote it by

$$
r=r(s)
$$

for $s \in\left(S_{0}, S\right)$. Let

$$
\chi(s)=\psi(r(s))
$$

Then (3.1) is transformed into

$$
\tilde{\mathcal{L}} \chi=-\ell \chi \quad \text { in }\left(S_{0}, S\right),
$$

where

$$
\tilde{\mathcal{L}} \chi=\frac{1 d}{\gamma d s}\left(r^{-4} p \rho \frac{d \chi}{d s}\right)-4 \frac{p^{\prime}}{r \rho} \chi
$$


Let $L^{2}\left(S_{0}, S\right)$ be the complex-valued $L^{2}$-space on $\left(S_{0}, S\right)$ with the standard inner product

$$
(\chi, \tilde{\chi}) \equiv \int_{S_{0}}^{S} \chi \tilde{\chi} d s
$$

It is clear that

$$
(\chi, \tilde{\chi})=\int_{R_{0}}^{R} \psi \tilde{\psi} W(r) d r \equiv(\psi, \tilde{\psi})_{w} .
$$

Here $(,)_{w}$ defines an inner product in space $L_{w}^{2}\left(R_{0}, R\right)$ by $(3.8)$.

Now we can prove $\tilde{\mathcal{L}}$ has limit-point-type singularity at $S$.

Lemma 3.2. If $S<\infty$, then $\tilde{\mathcal{L}}$ is limit-point type at $S$. Furthermore, for (EP), $\tilde{\mathcal{L}}$ is also limit-point type at $-\infty$.

Proof. From [2], it is known that $\tilde{\mathcal{L}}$ is the limit-point-type singularity at $S$ if we can find a solution pair $\{\ell, \chi\}$ for (3.6) in a neighborhood of $S$ such that $\chi$ is not $L^{2}$. This can be done as follows:

Since

$$
p=A C_{\gamma}^{\gamma} u^{q+1}
$$

and

$$
p^{\prime}=A(q+1) C_{\gamma}^{\gamma} u^{q} u^{\prime}
$$

we have

$$
\frac{p^{\prime}(r)}{r \rho(r)}=A(q+1) C_{\gamma}^{\gamma-1} \frac{u^{\prime}(r)}{r} .
$$

Hence (3.10) implies

$$
\lim _{r \rightarrow R} \frac{p^{\prime}(r)}{r \rho(r)}=A(q+1) C_{\gamma}^{\gamma-1} \frac{u^{\prime}(R)}{R}
$$

Fix $\hat{S} \in\left(S_{0}, S\right)$. For any real $\ell$, let $\chi$ be the real solution of the following initialvalue problem:

$$
\begin{gathered}
\tilde{\mathcal{L}} \chi=-\ell \chi \quad \text { in }(\hat{S}, S), \\
\chi(\hat{S})=0 \quad \text { and } \quad \chi^{\prime}(\hat{S})=1 .
\end{gathered}
$$

Denote $\hat{R}=r(\hat{S})$. Now (3.11) implies that there exists $\ell_{0}<0$ such that

$$
\ell_{0} r \rho(r)-4 p^{\prime}(r) \leq 0
$$

in $[\hat{R}, R]$. We claim that $\chi \notin L^{2}(\hat{S}, S)$ if $\ell \leq \ell_{0}$.

Indeed, if $\chi \in L^{2}(\hat{S}, S)$, then Lemma 3.1 and (3.8) imply that $\chi$ is bounded at $S$. From (3.12) and (3.13), we obtain

$$
\frac{1}{\gamma} \int_{\hat{S}}^{S} r^{-4} p \rho\left(\frac{d \chi}{d s}\right)^{2} d s=\ell \int_{\hat{S}}^{S} \chi^{2} d s-4 \int_{\hat{S}}^{S} p^{\prime} \chi^{2} d s .
$$


Now the left-hand side of (3.15) is positive and the right-hand side of (3.15) is nonpositive when $\ell \leq \ell_{0}$, a contradication. This implies that $\chi \notin L^{2}(\hat{S}, S)$ for $\ell \leq \ell_{0}$. Therefore, $\tilde{\mathcal{L}}$ is limit-point type at $S$.

For (EP), (3.10) and (1.7) imply that

$$
\lim _{r \rightarrow 0^{+}} \frac{p^{\prime}(r)}{r \rho(r)}=-\frac{A}{3}(q+1) C_{\gamma}^{\gamma-1} u^{q}(0) .
$$

Now using (3.11) and (3.16), we can choose $\ell_{0}<0$ such that $(3.14)$ holds in $(0, \hat{R})$. Let $\psi(r)=\chi(s(r))$; then Lemma 3.1 implies either

$$
\psi(0) \neq 0
$$

or

$$
|\psi(r)| \leq C r^{3} \quad \text { and } \quad\left|\psi^{\prime}(r)\right| \leq C r^{2}
$$

for some $C>0$. Now we can rule out the possibility of (3.18) when $\ell \leq \ell_{0}$. Indeed, if (3.18) holds, then

$$
0<\frac{1}{\gamma} \int_{-\infty}^{\hat{S}} r^{-4} p \rho\left(\frac{d \chi}{d s}\right)^{2} d s=\int_{-\infty}^{\hat{S}}\left(\ell-\frac{4 p^{\prime}}{r \rho}\right) \chi^{2} d s<0
$$

a contradication.

Hence we must have (3.17) when $\ell \leq \ell_{0}$, i.e., $\chi \notin L^{2}(-\infty, \hat{S})$. Therefore, $\tilde{\mathcal{L}}$ is a limit-point-type at $-\infty$. The proof is complete.

An immediate consequence of Lemma 3.2 is that $\tilde{\mathcal{L}}$ is self-adjoint. Indeed, we have the following result (for the proof, see [2]).

Corollary 3.3. For (EC) and (EPC), if $R<\infty$, let $\mathcal{D}_{1}$ be the set of all functions $\chi$ such that

(i) $\chi$ is differentiable and $\chi^{\prime}$ is absolutely continuous on $[0, \hat{S}]$ for any $\hat{S}<S$,

(ii) $\chi$ and $\tilde{\mathcal{L}} \chi \in L^{2}(0, S)$, and

(iii) $\chi(0)=0$.

Then $\tilde{\mathcal{L}}$ is self-adjoint, i.e.,

$$
(\tilde{\mathcal{L}} \chi, \hat{\chi})=(\chi, \tilde{\mathcal{L}} \hat{\chi})
$$

for all $\chi$ and $\hat{\chi}$ in $\mathcal{D}_{1}$.

Similarly, for $(\mathrm{EP})$, let $\mathcal{D}_{0}$ be the set of all functions $\chi$ such that

$(\mathrm{i})^{\prime} \chi$ is differentiable and $\chi^{\prime}$ is absolutely continuous over $(-\infty, \hat{S}]$ for any $\hat{S} \in$ $(-\infty, S)$ and

(ii) $)^{\prime} \chi$ and $\tilde{\mathcal{L}} \chi \in L^{2}(-\infty, S)$.

Then $\tilde{\mathcal{L}}$ is self-adjoint.

Furthermore, using Friedrichs' criteria, we can prove that $\tilde{\mathcal{L}}$ has only a discrete spectrum.

THEOREM 3.4. Let $u$ be a stationary solution of (EC), (EP), or (EPC) with $R<\infty$. The spectra of $\tilde{\mathcal{L}}$ consist of sequences of strictly increasing eigenvalues $\left\{\ell_{j}\right\}_{j=1}$ with associated eigenfunctions $\left\{\chi_{j}\right\}_{j=1}^{\infty}$ in $\mathcal{D}_{1}$ (or $\left.\mathcal{D}_{0}\right)$.

Proof. We first claim that no continuous spectrum comes out of $S$. Indeed, using (3.5), it can be verified that

$$
s(r)=S-c_{1}(R-r)^{q+1}+o\left((R-r)^{q+1}\right)
$$


for $r$ close to $R$, where $c_{1}>0$ depends on $R, u^{\prime}(R)$, and $\gamma$.

Let

$$
a(s)=\frac{1}{\gamma} r^{-4} p(r) \rho(r), \quad b(s)=\frac{4 p^{\prime}}{r \rho}, \quad \text { and } \quad c(s)=1 .
$$

(3.20) then implies

$$
a(s)=c_{2}(S-s)^{2-\varepsilon}+o\left((S-s)^{2-\varepsilon}\right),
$$

where $\varepsilon=\frac{1}{q+1}$ and $c_{2}>0$. Let

$$
h(s)=\int_{0}^{s} \frac{1}{a(\tau)} d \tau
$$

Then (3.21) implies

$$
h(s)=c_{3}(S-s)^{\varepsilon-1}+o\left((S-s)^{\varepsilon-1}\right)
$$

for $s$ close to $S$, where $c_{3}>0$.

Hence (3.21) and (3.22) imply that

$$
4 a h^{2}=c_{4}(S-s)^{\varepsilon}+o\left((S-s)^{\varepsilon}\right),
$$

where $c_{4}>0$. (3.11) now implies that $b(s)$ is bounded at $S$. Therefore, (3.23) implies that

$$
Z(s)=\frac{1}{c}\left\{b+\frac{1}{4 a h^{2}}\right\} \rightarrow+\infty
$$

as $s \rightarrow S$. By Proposition B.3 in Appendix B, no continuous spectrum comes out of $S$, and $\tilde{\mathcal{L}}$ is totally descrete in $R^{1}$. In particular, for (EC) and (EPC), the spectrum of $\tilde{\mathcal{L}}$ is a sequence of eigenvalues $\left\{\ell_{j}\right\}_{j=1}^{\infty}$ such that

$$
\lim _{j \rightarrow \infty} \ell_{j}=+\infty .
$$

For (EP), we also need to prove that (3.24) holds when $s \rightarrow-\infty$. From (3.5), we have

$$
s=-c_{0} r^{-1}+o\left(r^{-1}\right)
$$

for $r \rightarrow 0^{+}$, where $c_{0}>0$. Therefore,

$$
a(s)=c_{5} s^{4}+o\left(s^{4}\right)
$$

as $s \rightarrow-\infty$, where $c_{5}>0$. Let

$$
h(s)=\int_{s}^{0} \frac{1}{a(\tau)} d \tau .
$$

Then (3.27) implies

$$
h(s)=c_{6}(-s)^{-3}+o\left(s^{-3}\right)
$$

as $s \rightarrow-\infty$, where $c_{6}>0$. Hence (3.27) and (3.28) imply

$$
4 a h^{2}=c_{7} s^{-2}+o\left(s^{-2}\right)
$$


as $s \rightarrow-\infty$. (3.24) then follows from last equation and (3.16). Hence no continuous spectrum comes out from $-\infty$ for (EP). The proof is complete.

From Lemma $3.4, \tilde{\mathcal{L}}$ has only the real eigenvalue $\ell$. Therefore, $\lambda$ is either real or purely imaginary for any eigenvalue $\lambda$.

From these observations, we then introduce the following notion of stability.

DEFINITION 3.5. Let u be a ball-type stationary solution of (EC), (EP), or (EPC), and let $\left\{\ell_{j}\right\}_{j=1}^{\infty}$ be the associated eigenvalues of $\tilde{\mathcal{L}}$ given in Theorem 3.4. $u$ is then called neutrally stable if $\ell_{1}>0$ (i.e., $\lambda_{1}= \pm i \sqrt{ } \ell_{1}$ is purely imaginary), is called unstable if $\ell_{1}<0$ (i.e., $\lambda_{1}= \pm \sqrt{ }\left|\ell_{1}\right|$ is real), and is called marginally stable if $\ell_{1}=0$.

A similar definition can also be given for ground-state- and singularity-type solutions.

Remark 3.6. From Lemma 3.1 and Theorem 3.4, if $\psi$ is an eigenfunction, then $\psi(R)$ is bounded. Furthermore, for (EP), $\psi(r)=O\left(r^{3}\right)$ as $r \rightarrow 0^{+}$. Moreover, the least eigenvalue $\ell_{1}$ can be obtained by a variational method; see, e.g., [3]. Indeed, for (EC) or (EPC), we have

$$
\ell_{1}=\inf \left\{\frac{Q(\psi)}{I(\psi)}: \psi(1)=0 \quad \text { and } \quad \psi \in C^{1}[1, R]\right\}
$$

where

$$
Q(\psi)=\int_{1}^{R}\left\{r^{-2} p(r) \psi^{\prime 2}(r)+\frac{4}{\gamma} r^{-3} p^{\prime}(r) \psi^{2}(r)\right\} d r
$$

and

$$
I(\psi)=\frac{1}{\gamma} \int_{1}^{R} r^{-2} \rho(r) \psi^{2}(r) d r .
$$

A similar formulation also holds for (EP) with $\psi(r)=O\left(r^{3}\right)$ as $r \rightarrow 0^{+}$.

The following comparison lemma is very useful for testing the stability of stationary solutions.

Lemma 3.7. Let u be a BC-type stationary solution for (EC) or (EPC). Then the following hold:

(i) If there exists a $\tilde{\psi} \in C^{2}([1, R])$ with $\tilde{\psi}(1)=0, \tilde{\psi}>0$ in $(1, R]$, that satisfies

$$
L_{0} \tilde{\psi} \leq 0(\text { but not } \equiv) \quad \text { in }(1, R),
$$

then $u$ is neutrally stable.

(ii) If there exists a $\psi \in C^{2}([1, R])$ with $\psi(1)=0, \psi>0$ in $(1, R]$, that satisfies

$$
L_{0} \psi \geq 0(\text { but not } \equiv) \quad \text { in }(1, R) \text {, }
$$

then $u$ is unstable.

A similar result also holds for a B-type stationary solution u for (EP) provided the comparison function $\tilde{\psi}($ or $\psi)$ satisfies $\tilde{\psi}($ or $\psi) \in L_{w}^{2}(0, R)$ and $\mathcal{L} \tilde{\psi}($ or $\mathcal{L} \psi) \in$ $L_{w}^{2}(0, R)$.

Proof. Let $\psi_{1}>0$ in $\left(R_{0}, R\right)$ be the associated eigenfunction with respect to $\ell_{1}$ in (3.20). If there is a $\tilde{\psi}$ that satisfies all conditions in (i), then it is easy to see that $\mathcal{L} \tilde{\psi} \leq 0$ in $(1, R)$, which implies that

$$
0=\int_{1}^{R}\left(\tilde{\psi} \mathcal{L} \psi_{1}-\psi_{1} \mathcal{L} \tilde{\psi}\right) d r>-\ell_{1} \int_{1}^{R} W \tilde{\psi} \psi_{1} d r
$$


Therefore, $\ell_{1}>0$. This proves (i). (ii) and the cases for (EP) can be proved analogously. The proof is complete.

4. Stability results. In this section, we shall use the methods developed in the preceding section to study the stability of various stationary solutions. We begin with ball-type solutions, proceed to ground-state solutions, and finally conclude with singular solutions.

4.1. Ball-type solutions. We first introduce an auxiliary operator $\tilde{L}$, defined as

$$
\tilde{L} \psi \equiv \psi^{\prime \prime}-\frac{(q+3)}{r} \psi^{\prime}+\frac{4 q}{r^{2}} \psi .
$$

$\tilde{L}$ is closed related to $L_{0}$, as can be seen from the following:

$$
L_{0} \psi=\tilde{L} \psi+\left\{(1+q) \psi^{\prime}-\frac{4 q}{r} \psi\right\}\left(\frac{u^{\prime}}{u}+\frac{1}{r}\right) .
$$

The following results for operator $\tilde{L}$ are very useful in constructing the comparison functions $\tilde{\psi}$ and $\psi$ according to Lemma 3.7.

Lemma 4.1. For any $q>1$, we have $\tilde{L}\left(r^{4}\right)=0$ and $\tilde{L}\left(r^{q}\right)=0$. Moreover, if $q=4$, we also have $\tilde{L}\left(r^{4} \log r\right)=0$.

Furthermore, if we let (i) $\tilde{\psi}=r^{4}-r^{q}$ if $q \in(1,4)$, (ii) $\tilde{\psi}=r^{4} \log r$ if $q=4$, and (iii) $\tilde{\psi}=r^{q}-r^{4}$ if $q \in(4, \infty)$, then we have (a) $\tilde{L} \tilde{\psi}=0$ for $r>1$, (b) $\tilde{\psi}(1)=0$, and (c) the following:

$$
(1+q) \tilde{\psi}^{\prime}-\frac{4 q}{r} \tilde{\psi}>0 \quad \text { for } \quad r>1
$$

Proof. The computations are straightforward, so we verify only the last inequality and omit the others. Indeed, for $q \neq 4$,

$$
(1+q)\left(r^{q}-r^{4}\right)^{\prime}-\frac{4 q}{r}\left(r^{q}-r^{4}\right)=q(q-3) r^{q-1}-4 r^{3},
$$

and for $q=4$,

$$
(1+q)\left(r^{4} \log r\right)^{\prime}-\frac{4 q}{r}\left(r^{4} \log r\right)=4 r^{3} \log r+5 r^{3} .
$$

The result follows.

Next, it is easy to verify the following lemma, so we omit the proof.

Lemma 4.2. If $u>0$ in $(1, R)$ and satisfies the equation

$$
u^{\prime \prime}+\frac{2}{r} u^{\prime}+f(u)=0 \quad \text { for } r>1,
$$

then

$$
\frac{d}{d r}\left(\frac{u^{\prime}}{u}+\frac{1}{r}\right)=-\frac{1}{r^{2} u^{2}}\left\{\left(r u^{\prime}+u\right)^{2}+r^{2} u f(u)\right\} .
$$

In particular, if $u(\cdot, \alpha, \mu)$ is a solution of (1.6) or (1.8), then $\alpha \leq \mu$ implies

$$
\left(\frac{u^{\prime}}{u}+\frac{1}{r}\right)<0 \quad \text { in }(1, R) .
$$

We can now establish the stability results for (EC) and (EPC) when $\alpha \leq \mu$. 


\section{THEOREM 4.3 .} stable.

(i) For any $q>1, \mu>0$, and $R>1$, the solution $u_{R, \mu}$ of (EC) is neutrally

(ii) For any $q>1$, let $u(\cdot, \alpha, \mu)$ be the solution of (EPC). Then $u(\cdot, \alpha, \mu)$ is neutrally stable if $\alpha \leq \mu$.

Proof. It is not difficult to verify $R(\alpha, \mu)<\infty$ when $\alpha \leq \mu$ in (ii). Let $\tilde{\psi}$ be given as in Lemma 4.1. Then for both (i) and (ii), Lemmas 4.1 and 4.2 imply $L \tilde{\psi}<0$ in $(1, R)$.

Thus Lemma 3.7 implies that $u_{R, \mu}$ and $u(\cdot, \alpha, \mu)$ with $0<\alpha \leq \mu$ are neutrally stable. The proof is complete.

We can also establish other stability results for (EPC) by choosing appropriate comparison functions and applying Lemma 3.7. For example, we can prove the following theorem.

THEOREM 4.4. For (EPC), we have the following:

(i) If $q \in(1,3]$, then all BC-type solutions are neutrally stable.

(ii) For any $q>1$, there is $R_{q}>1$ such that $u$ is neutrally stable whenever the first zero $R$ of $u$ is less than $R_{q}$.

Proof. (i) It is known that $R(\alpha, \mu)<\infty$ for any $\alpha>0$ and $\mu>0$ when $q \in(1,3]$; see, e.g., [18]. Let $\tilde{\psi}=r^{3}-1$. Then $\tilde{\psi}(1)=0, \tilde{\psi}>0$ in $(0, \infty)$, and

$$
L_{0} \tilde{\psi}=\left\{\frac{u^{\prime}}{u}(3-q) r^{2}+\frac{4 q}{r}\right\},
$$

which is negative in $(0, \infty)$ if $q \in(1,3]$. Thus by Lemma $3.7(\mathrm{i}), u$ is neutrally stable.

(ii) Let $\tilde{\psi}=\log r$. Then $\tilde{\psi}(1)=0, \tilde{\psi}>0$ in $(1, \infty)$, and

$$
L_{0} \tilde{\psi}=-3 r^{-2}+\frac{1}{r} \frac{u^{\prime}}{u}\{(1+q)-4 q \log r\} .
$$

Therefore, $L_{0} \tilde{\psi}<0$ in $(1, R)$ if $R \leq R_{q} \equiv \exp \left(\frac{1+q}{4 q}\right)$. The result also follows from Lemma 3.7(i). The proof is complete.

Remark 4.5. By picking a comparison function $\tilde{\psi}$ different from $\log r$ in Theorem 4.4(ii), we can also obtain another $\tilde{R}_{q}$, which ensures that $u$ is neutrally stable when $R \leq \tilde{R}_{q}$.

By choosing an appropriate comparison function, we obtain the following stability results for $(\mathrm{EP})$.

THEOREM 4.6. For (EP), we have the following:

(i) If $q \in(1,3)$, then any B-type solution is neutrally stable.

(ii) If $q=3$, then any B-type solution is marginally stable.

(iii) If $q \in(3,5)$, then any B-type solution is unstable.

Proof. Let $\tilde{\psi}=r^{3}$. Then $\tilde{\psi}(0)=\tilde{\psi}^{\prime}(0)=0$ and $\tilde{\psi}>0$ in $(0, \infty)$. Furthermore, we have

$$
L_{0} \tilde{\psi}=(3-q) r^{2} \frac{u^{\prime}}{u} \text {. }
$$

Hence the result follows by Lemma 3.7. The proof is complete.

Proof of Theorem 1.1. Combining the results from Theorems 4.3, 4.4, and 4.6, we obtain Theorem 1.1. 
4.2. Ground-state solutions. From section 2, we know that if a ground-statetype solution $u$ has a finite total mass, then it is necessary that $u$ have a fast decay rate, i.e.,

$$
\lim _{r \rightarrow \infty} r u(r)=m \in(0, \infty) .
$$

In this section, we will prove that the linearized operator $\mathcal{L}$ associated with $u$ has a continuous spectrum $(0, \infty)$. Therefore, $u$ cannot be neutrally stable. In fact, it is either marginally stable or unstable.

Lemma 4.7. If $u$ is a $G$-or GC-type solution and satisfies (4.5), then the linearized operator $\mathcal{L}$ of $u$ is discrete below 0 and has a continuous spectrum $(0, \infty)$.

Proof. In Lemma 3.4, we have shown that no continuous spectrum comes from $r=0$ for $\mathcal{L}$ in (EP). Therefore, we need only study $\mathcal{L}$ as $r \rightarrow \infty$. We may assume that $m=1$ in (4.5). We then have

$$
p(r)=\tilde{A} r^{-1-q}+o\left(r^{-1-q}\right)
$$

and

$$
p^{\prime}(r)=-(1+q) \tilde{A} r^{-q}+o\left(r^{-q}\right)
$$

as $r \rightarrow \infty$, where $\tilde{A}=A C_{\gamma}^{\gamma}$. As before, we have the following asymptotic expansions for the coefficients of $\mathcal{L}$ as $r \rightarrow \infty$ :

$$
\begin{gathered}
a(r)=r^{-2} p(r)=\tilde{A} r^{-3-q}+o\left(r^{-3-q}\right), \\
b(r)=\frac{4}{\gamma} r^{-3} p^{\prime}(r)=-4 q \tilde{A} r^{-5-q}+o\left(r^{-5-q}\right),
\end{gathered}
$$

and

$$
c(r)=\frac{1}{\gamma} r^{-2} \rho(r)=\hat{A} r^{-2-q}+o\left(r^{-2-q}\right)
$$

as $r \rightarrow \infty$, where $\hat{A}>0$ is a constant. Therefore, for large fixed $\hat{r}$, we have

$$
h(r)=\int_{\hat{r}}^{r} \frac{d \tau}{a(\tau)}=\{\tilde{A}(4+q)\}^{-1} r^{4+q}+o\left(r^{4+q}\right)
$$

as $r \rightarrow \infty$.

We claim that

$$
Z(r)=\frac{1}{c(r)}\left\{b(r)+\frac{1}{4 a(r) h^{2}(r)}\right\} \rightarrow 0 \quad \text { as } r \rightarrow \infty .
$$

Indeed, it is clear that

$$
4 a(r) h^{2}(r)=4 \tilde{A}^{-1}(4+q)^{-2} r^{q+5}+o\left(r^{5+q}\right) \quad \text { as } r \rightarrow \infty .
$$

Therefore, we have

$$
\begin{aligned}
b(r)+\frac{1}{4 a(r) h^{2}(r)} & =\tilde{A}\left\{\frac{(4+q)^{2}}{4}-4 q\right\} r^{-5-q}+o\left(r^{-5-q}\right) \\
& =\frac{\tilde{A}}{4}(q-4)^{2} r^{-5-q}+o\left(r^{-5-q}\right) .
\end{aligned}
$$


Hence

$$
Z(r)=A^{*} r^{-3}(q-4)^{2}+o\left(r^{-3}\right)
$$

for some $A^{*}>0$. (4.6) follows. Now by Proposition B.3 (II)-(III) in Appendix B, the linearized operator $\mathcal{L}$ of $u$ has a continuous spectrum $(0, \infty)$ and is descrete below 0 . The proof is complete.

An immediate consequence of Lemma 4.7 is the following theorem for groundstate-type stationary solutions.

THEOREM 4.8. Any ground-state-type solution of (EC), (EP), or (EPC) is either marginally stable or unstable.

4.3. Singular solutions. In this section, we will continuously apply Friedrichs' criteria to study the stability of singularity-type solutions. We know that if $q \in(3,5)$ and $u$ is a singular solution of (EP) with finite total mass, then $u$ has a weak singularity at $r=0$, i.e.,

$$
\lim _{r \rightarrow 0^{+}} r^{\sigma} u(r)=m \in(0, \infty)
$$

As in section 4.2, we are interested in the limit of $Z(r)$ as $r \rightarrow 0^{+}$. (4.7) now implies the following expansions:

$$
\begin{gathered}
a(r)=r^{-2} p(r)=\tilde{A} r^{-\sigma(q+1)-2}+o\left(r^{-\sigma(q+1)-2}\right), \\
b(r)=\frac{4}{\gamma} r^{-3} p^{\prime}(r)=-4 \sigma q \tilde{A} r^{-\sigma(q+1)-4}+o\left(r^{-\sigma(q+1)-4}\right),
\end{gathered}
$$

and

$$
c(r)=\hat{A} r^{-2-\sigma q}+o\left(r^{-2-\sigma q}\right)
$$

as $r \rightarrow 0^{+}$for some positive constants $\tilde{A}$ and $\hat{A}$.

Therefore,

$$
h(r)=\int_{0}^{r} \frac{d s}{a(s)}=\tilde{A}^{-1}\{3+\sigma(q+1)\}^{-1} r^{\sigma(q+1)+3}
$$

with $h(0)=0$.

It is straightfoward to compute

$$
\begin{aligned}
Z(r) & =A^{*} r^{-\sigma-2}\left\{\frac{1}{4}[3+\sigma(q+1)]^{2}-4 \sigma q\right\}+o\left(r^{-\sigma-2}\right) \\
& ={ }^{*}{ }^{*} r^{-\sigma-2}\left\{4 \sigma^{2}+4 \sigma-7\right\}+o\left(r^{-\sigma-2}\right)
\end{aligned}
$$

for some positive constant $A^{*}$.

Hence we obtain the following lemma.

LEMma 4.9. Let $u$ be a singular solution of (EP) satisfying (4.7). Then we have the following:

(i) if $q \in\left(3, q^{+}\right)$, then $\lim _{r \rightarrow 0^{+}} Z(r)=+\infty$;

(ii) if $q=q^{+}$, then $\lim _{r \rightarrow 0^{+}} Z(r)=0$;

(iii) if $q \in\left(q^{+}, 5\right)$, then $\lim _{r \rightarrow 0^{+}} Z(r)=-\infty$; 
and

$$
\Omega_{0}=\int_{0}^{\hat{r}}\left\{\begin{array}{c}
c(r) \\
-a(r) Z(r)
\end{array}\right\}^{\frac{1}{2}} d r<\infty .
$$

Therefore, by applying Theorem 4.6(iii), Lemma 4.9, and Proposition B.3, we obtain the following theorem for singularity-type solutions.

THEOREM 4.10. For problem (EP), we have the following:

(i) If $q \in\left(3, q^{+}\right)$, then any SB-type solution is unstable and has no continuous spectrum. Any $S G$-type solution is also unstable but has a continuous spectrum $(0, \infty)$.

(ii) If $q=q^{+}$, then any $S B$-type and $S G$-type solution is unstable and has a continuous spectrum $(0, \infty)$.

(iii) If $q \in\left(q^{+}, 5\right)$, then any $S B$-type and $S G$-type solution is unstable, and there is a sequence of pure imaginary eigenvalues $\left\{\lambda_{k}\right\}$ such that $\lim _{k \rightarrow \infty} \lambda_{k}^{2}=-\infty$.

Proof. For any $q \in(3,5)$ and for an SB-type solution $u$, choose $\tilde{\psi}=r^{3}$. Then we have

$$
L \tilde{\psi}=(3-q) r^{2} \frac{u^{\prime}}{u}>0 .
$$

Therefore, by modifying the proof of Lemma 3.5, we can prove that $u$ is unstable. The remaining results follow from Lemma 4.9 and Proposition B.3. The details of the proof are omitted and the proof is complete.

5. Effects of viscosity. In this section, we shall study the effect of viscosity on the stability problem of stationary solutions. From equation (1.2), it is clear that stationary solutions for inviscid flow are also solutions for viscous flow. As we have seen in the previous sections, the best possibilities for stationary solutions are neutrally stable in the inviscid case. It is known that neutral stability is very sensitive to disturbances. Therefore, we need to know what effect viscosity has on neutrally stable stationary solutions.

Since the gaseous mass is not confined from outside, its outer surface is a free surface maintained by the attraction of the core and its own gravitational forces. Presumably, the surface of the gas should be very sensitive to a direct disturbance of it. In this section, we show that this is the case, as mentioned in Theorem 1.2.

When viscosity its present, the linearized equation is

$$
\mathcal{L} \psi=\lambda^{2} W \psi-\lambda \nu \hat{\mathcal{L}} \psi,
$$

where

$$
\hat{\mathcal{L}} \psi \equiv \frac{1}{\gamma}\left(r^{-2} \psi^{\prime}\right)^{\prime}
$$

or, equivalently,

$$
\left\{r^{-2}\left(p(r)+\lambda \frac{\nu}{\gamma}\right) \psi^{\prime}\right\}^{\prime}-\frac{1}{\gamma}\left\{4 r^{-3} p^{\prime}(r)+\lambda^{2} r^{-2}\right\} \psi=0 .
$$

When $\nu>0$, the eigenvlaue equation (5.1) is linear for $\psi$ but quadratic for $\lambda$, which is different from ordinary eigenvalue problems. Indeed, if $\nu=0$ in (5.1), then (5.1) is linear for $\ell=-\lambda^{2}$. Since the coefficients of $\mathcal{L}, \hat{\mathcal{L}}$, and $W$ are real, it is easy to see that if $\{\lambda, \psi\}$ is a solution of (5.1), then its conjugate $\{\lambda, \psi\}$ is also a solution. 
This property does not affect the stability, which depends on the sign of $\operatorname{Re} \lambda$ in the stationary solution.

In this section, we concentrate on the effects of viscosity and boundary disturbances. Therefore, we restrict our study to ball-type solutions which are neutrally stable. The problems of unstable stationary solutions, ground-state solutions, and singularity-type solutions will be left for future study.

We first consider (EC) and (EPC) and then continue by studying (EP).

Let

$$
\lambda^{*}=\frac{\gamma}{\nu} P\left(R_{0}\right) .
$$

When $R_{0}=1$, we will prove that (5.1) is regular on $[1, R]$ when $\lambda \notin\left[-\lambda^{*}, 0\right]$. Indeed, for $\lambda \neq-\lambda^{*}$, let $\psi(\cdot, \lambda)=\psi(\cdot, \lambda, \nu)$ be the solution of (5.3) that satisfies the initial conditions

$$
\psi(1,0)=0
$$

and

$$
\psi^{\prime}(1, \lambda)=1
$$

We can then prove the following result.

LEMma 5.1. Let $u$ be a BC-type stationary solution of (EC) or (EPC). If $\lambda \notin$ $\left[-\lambda^{*}, 0\right]$, then $\psi(\cdot, \lambda)$ is $C^{2}$ on $[1, R]$ and is analytic in $\lambda \in \mathbf{C}-\left[-\lambda^{*}, 0\right]$. Furthermore, if $\lambda \in\left(-\lambda^{*}, 0\right)$, then either $\psi(\cdot, \lambda)$ is bounded at $r=\hat{r}$ or $|\psi(r, \lambda)|$ grows like $|\log | r-\hat{r} \|$ as $r \rightarrow \hat{r}$, where $\hat{r} \in(1, R)$ satisfies $p(\hat{r})+\lambda_{\gamma}^{\nu}=0$. If $\lambda=-\lambda^{*}$, then any nontrivial solution $\psi$ of (5.3) is unbounded in a neighborhood of $r=1$. The case in which $\lambda=0$ was studied in Lemma 3.1 .

Proof. Let $\lambda=\lambda_{1}+i \lambda_{2}$ and $\psi=\psi_{1}+i \psi_{2}$ in (5.3) and denote

$$
\begin{array}{ll}
a=r^{-2}\left(p+\lambda_{1} \frac{\nu}{\gamma}\right), & b=\lambda_{2} \frac{\nu}{\gamma} r^{-2}, \\
c=\frac{1}{\gamma}\left\{4 r^{-3} p^{\prime}+r^{-2}\left(\lambda_{1}^{2}-\lambda_{2}^{2}\right)\right\}, & d=\frac{2}{\gamma} \lambda_{1} \lambda_{2} r^{-2} .
\end{array}
$$

Then it is clear that $a^{2}(\hat{r})+b^{2}=0$ for some $\hat{r} \in[1, R]$ if and only if $\lambda_{2}=0$ and $\lambda_{1} \in\left[-\lambda^{*}, 0\right]$. In this case, $p(\hat{r})+\lambda_{1} \frac{\nu}{\gamma}=0$.

Now (5.3) can be written as the following system of equations:

$$
\begin{aligned}
& \left(a \psi_{1}^{\prime}-b \psi_{2}^{\prime}\right)^{\prime}=c \psi_{1}-d \psi_{2}, \\
& \left(b \psi_{1}^{\prime}+a \psi_{2}^{\prime}\right)^{\prime}=d \psi_{1}+c \psi_{2} .
\end{aligned}
$$

For $\lambda \notin\left[-\lambda^{*}, 0\right]$, denote

$$
\tilde{\psi}_{1}=a \psi_{1}-b \psi_{2} \quad \text { and } \quad \tilde{\psi}_{2}=b \psi_{1}+a \psi_{2} .
$$

We then have

$$
\begin{aligned}
& \psi_{1}=\left(a \tilde{\psi}_{1}+b \tilde{\psi}_{2}\right)\left(a^{2}+b^{2}\right)^{-1}, \\
& \psi_{2}=\left(-b \tilde{\psi}_{1}+a \tilde{\psi}_{2}\right)\left(a^{2}+b^{2}\right)^{-1} .
\end{aligned}
$$


By a straightforward but lengthy computation on (5.7), we obtain the following system of equations for $\tilde{\psi}_{1}$ and $\tilde{\psi}_{2}$ :

$$
\begin{aligned}
& \tilde{\psi}_{1}^{\prime \prime}=\tilde{A} \tilde{\psi}_{1}^{\prime}+\tilde{B} \tilde{\psi}_{1}+\tilde{C} \tilde{\psi}_{2}^{\prime}+\tilde{D} \tilde{\psi}_{2}, \\
& \tilde{\psi}_{2}^{\prime \prime}=\tilde{A} \tilde{\psi}_{2}^{\prime}+\tilde{B} \tilde{\psi}_{2}-\tilde{C} \tilde{\psi}_{1}^{\prime}-\tilde{D} \tilde{\psi}_{2},
\end{aligned}
$$

where

$$
\tilde{a}=a\left(a^{2}+b^{2}\right)^{-1}, \quad \tilde{b}=b\left(a^{2}+b^{2}\right)^{-1},
$$

and

$$
\begin{aligned}
& \tilde{A}=a^{\prime} \tilde{a}+b^{\prime} \tilde{b} \\
& \tilde{B}=\left(a^{\prime \prime}+c\right) \tilde{a}+\left(b^{\prime \prime}+d\right) \tilde{b}+a^{\prime} \tilde{a}^{\prime}+b^{\prime} \tilde{b}^{\prime}, \\
& \tilde{C}=a^{\prime} \tilde{b}-b^{\prime} \tilde{a} \\
& \tilde{D}=\left(a^{\prime \prime}+c\right) \tilde{b}-\left(b^{\prime \prime}+d\right) \tilde{s}+a^{\prime} \tilde{b}^{\prime}-b^{\prime} \tilde{a}^{\prime} .
\end{aligned}
$$

Since the coefficients of (5.10) are continuous on $[1, R]$, then $\tilde{\psi}_{1}^{\prime}$ and $\tilde{\psi}_{2}^{\prime}$ are $C^{2}$ on $[1, R]$ and analytic in $\lambda \in \mathbf{C}-\left[-\lambda^{*}, 0\right]$. Hence $\psi_{1}$ and $\psi_{2}$ have the same properties as $\tilde{\psi}_{1}$ and $\tilde{\psi}_{2}$. This proves the first part of the lemma.

To study $\lambda \in\left(-\lambda^{*}, 0\right)$, we write $(5.3)$ as

$$
\psi^{\prime \prime}+\left\{\frac{1}{r-\hat{r}}+g(r)\right\} \psi^{\prime}+\frac{1}{r-\hat{r}} f(r) \psi=0 \quad \text { for } r<\hat{r},
$$

where $g$ and $f$ are analytic at $\hat{r}$. Hence $\hat{r}$ is a regular singular point. Therefore, by a standard theorem (see, e.g., [2]), this implies that $\psi$ either is bounded at $\hat{r}$ or grows logarithmically at $\hat{r}$.

Finally, if $\lambda=-\lambda^{*}$, then $p(1)=\lambda^{*}{ }_{\gamma}$. Let $s=r-1$; then (5.3) can be written as

$$
\psi^{\prime \prime}+\left(\frac{2}{s}+g\right) \psi^{\prime}+\left(\frac{c_{2}}{s^{2}}+\frac{c_{1}}{s}+f\right) \psi=0 \quad \text { for } s>0,
$$

where $g$ and $f$ are continuous at $s=0$ and $c_{2}>0$.

Let

$$
\mu_{1}=\frac{1}{2}\left(-1+\sqrt{ } 1-4 c_{2}\right) \quad \text { and } \quad \mu_{2}=\frac{1}{2}\left(-1-\sqrt{ } 1-4 c_{2}\right) .
$$

If $\mu_{1} \neq \mu_{2}$, then $\psi$ behaves asymptotially like $s^{\mu_{1}}$ or $s^{\mu_{2}}$ as $s \rightarrow 0^{+}$. If $\mu_{1}=\mu_{2}=-\frac{1}{2}$, then $|\psi(s)|$ behaves asymptotically like $s^{-\frac{1}{2}}$ or $s^{-\frac{1}{2}}|\log s|$ as $s \rightarrow 0^{+}$. In any case, $\psi$ is unbounded at $s=0$. The case in which $\lambda=0$ was studied in Lemma 3.1. The proof is complete.

Considering (1.13) and Lemma 5.1, we introduce the following notion.

Definition 5.2. For $\lambda \notin\left[-\lambda^{*}, 0\right], \psi(\cdot, \lambda)$ is called a stable mode if $\operatorname{Re} \lambda<0$, an unstable mode if $\operatorname{Re} \lambda>0$, and a marginally stable mode if $\operatorname{Re} \lambda=0$.

In the following, we shall study the relationship between the sign of $\operatorname{Re} \lambda$ and $\psi(R, \lambda)$, i.e., how the disturbance of the gas surface influences the stability of the stationary solution $u$.

Since $\psi(\cdot, \lambda)$ is $C^{2}$ in $[1, R]$ for $\lambda \notin\left[-\lambda^{*}, 0\right], \psi(R, \lambda)$ and $\psi^{\prime}(R, \lambda)$ satisfies homogeneous boundary conditions at $R$, i.e.,

$$
a_{j} \psi_{j}^{\prime}(R)+b_{j} \psi_{j}(R)=0,
$$


where $\psi_{1}=\operatorname{Re} \psi$ and $\psi_{2}=\operatorname{Im} \psi, a_{j}=a_{j}(\lambda)$ and $b_{j}=b_{j}(\lambda)$ are analytic in $\lambda \notin\left[-\lambda^{*}, 0\right]$ for $j=1,2$.

When $a_{j} \neq 0$, denote

$$
\kappa_{j}(\lambda)=\frac{b_{j}(\lambda)}{a_{j}(\lambda)}
$$

Then (5.13) can be written as

$$
\frac{\psi_{j}^{\prime}(R)}{\psi_{j}(R)}=-\kappa_{j}
$$

When $a_{j}=0$, i.e., $\psi_{j}$ satisfies the Dirichlet boundary condition $\psi_{j}(R)=0$, we adopt the convention $\kappa_{j}=+\infty$.

We can now introduce the notion of the stability of stationary solutions with respect to the boundary conditions (5.5) and (5.15) (or (5.13)).

Definition 5.3. Let $u$ be a BC-type stationary solution for (EC) or (EPC). Then $u$ is called stable with respect to (5.5) and (5.15) if any eigenvalue $\lambda$ of (5.1), (5.5), and (5.15) satisfies $\operatorname{Re} \lambda<0 . u$ is called unstable if there is an eigenvalue $\tilde{\lambda}$ of (5.1), (5.5), and (5.15) such that $\operatorname{Re} \tilde{\lambda}>0 . u$ is called marginally stable if any eigenvalue $\lambda$ of (5.1), (5.5), and (5.15) satisfies $\operatorname{Re} \lambda \leq 0$ and equality holds for some $\tilde{\lambda}$.

The stability problem with respect to boundary condition (5.15) can also be studied by making the following observation:

Denote

$$
\begin{gathered}
\mathbf{C}^{+}=\{\lambda \in \mathbf{C}: \operatorname{Re} \lambda>0\}, \quad \mathbf{C}^{-}=\{\lambda \in \mathbf{C}: \operatorname{Re} \lambda<0\} \\
\text { and } \mathbf{C}^{0}=\{\lambda \in \mathbf{C}: \operatorname{Re} \lambda=0\} .
\end{gathered}
$$

For any stationary solution $u$ and any

$$
\left(\kappa_{1}, \kappa_{2}\right) \in \mathbf{R}^{2} \equiv \mathbf{R}^{2} \cup\left\{\left(k_{1}, \infty\right): \kappa_{1} \in \mathbf{R}^{1}\right\} \cup\left\{\left(\infty, k_{2}\right): \kappa_{2} \in \mathbf{R}^{1}\right\} \cup\{(\infty, \infty)\},
$$

denote by $\sigma\left(\kappa_{1}, \kappa_{2}\right)$ the set of eigenvalues of (5.1), (5.5), and (5.15). Then define

$$
\begin{gathered}
K_{s}=K_{s}(u) \equiv\left\{\left(\kappa_{1}, \kappa_{2}\right): \sigma\left(\kappa_{1}, \kappa_{2}\right) \subset \mathbf{C}^{-}\right\}, \\
K_{u}=K_{u}(u) \equiv\left\{\left(\kappa_{1}, \kappa_{2}\right): \sigma\left(\kappa_{1}, \kappa_{2}\right) \cap \mathbf{C}^{+} \neq \phi\right\},
\end{gathered}
$$

and

$$
K_{m}=K_{m}(u)=\left\{\left(\kappa_{1}, \kappa_{2}\right): \sigma\left(\kappa_{1}, \kappa_{2}\right) \cap \mathbf{C}^{+}=\phi \quad \text { and } \quad \sigma\left(\kappa_{1}, \kappa_{2}\right) \cap \mathbf{C}^{0} \neq \phi\right\} .
$$

From Lemma 5.1, we know that any one of $K_{s}, K_{u}$, and $K_{m}$ is nonempty. Hence the stability of $u$ with respect to a given $\left(\kappa_{1}, \kappa_{2}\right)$ is equivalent to deciding to which set- $K_{s}, K_{u}$, or $K_{m}-\left(\kappa_{1}, \kappa_{2}\right)$ belongs. In general, for a given $u$, it is not easy to completely identify $K_{s}, K_{u}$, and $K_{m}$. However, we shall find some subsets of $K_{s}$ and $K_{u}$ that will give us sufficient conditions to determine whether $u$ is stable or unstable with respect to given $\left(\kappa_{1}, \kappa_{2}\right)$.

We first prove the following stability result. 
THEOREM 5.4. Let $u$ be a neutrally stable BC-type stationary solution of (EC) or $(\mathrm{EPC})$ when $\nu=0$. Then for any $\nu>0$, we have

$$
\left\{\left(\kappa_{1}, \kappa_{2}\right): \kappa_{1} \geq 0, \quad \kappa_{2} \geq 0\right\} \subset K_{s}(u),
$$

i.e., $u$ is stable if

$$
\kappa_{1} \geq 0 \quad \text { and } \quad \kappa_{2} \geq 0
$$

or, equivalently, if

$$
\psi_{j}^{\prime}(R) \psi_{j}(R) \leq 0
$$

for $j=1,2$.

Proof. Since $u$ is assumed to be neutrally stable when $\nu=0,0$ is not an eigenvalue of (5.1), (5.5), and (5.15). If $\lambda \in\left(-\lambda^{*}, 0\right)$, then there is nothing to prove. Hence we consider the case where $\lambda \notin\left[-\lambda^{*}, 0\right)$ and is an eigenvalue with respect to (5.15) such that $\left(\kappa_{1}(\lambda), \kappa_{2}(\lambda)\right)$ satisfies $(5.17)$. We must prove that

$$
\operatorname{Re} \lambda<0 .
$$

Indeed, multiply (5.1) by $\psi$ and then integrate from 1 to $R$; $\lambda$ satisfies

$$
a \lambda^{2}+b \lambda+c=0,
$$

where

$$
\begin{gathered}
a=\frac{1}{\gamma} \int_{1}^{R} r^{-2} \rho(r)\left(\psi_{1}^{2}+\psi_{2}^{2}\right) d r>0, \\
b=-\nu \int_{1}^{R} \psi \hat{\mathcal{L}} \psi d r, \quad \text { and } \quad c=-\int_{1}^{R} \psi \mathcal{L} \psi d r .
\end{gathered}
$$

Since $u$ is assumed to be neutrally stable when $\nu=0$, we have $\ell_{1}>0$ in (3.1). Moreover, $\psi$ is $C^{2}$ on $[1, R]$. Hence Remark 3.6 implies that

$$
c>0 .
$$

Now let

$$
b=b_{1}+i b_{2},
$$

where

$$
\begin{aligned}
b_{1} & =-\nu \int_{1}^{R}\left(\psi_{1} \hat{\mathcal{L}} \psi_{1}+\psi_{2} \hat{\mathcal{L}} \psi_{2}\right) \\
& =\nu\left\{\sum_{j=1}^{2} \int_{1}^{R} r^{-2}\left(\psi_{j}^{\prime}\right)^{2}-\sum_{j=1}^{2} R^{-2} \psi_{j}^{\prime}(R) \psi_{j}(R)\right\}
\end{aligned}
$$

and

$$
\begin{aligned}
b_{2} & =-\nu \int_{1}^{R}\left(\psi_{1} \hat{\mathcal{L}} \psi_{2}-\psi_{2} \hat{\mathcal{L}} \psi_{1}\right) \\
& =\frac{\nu}{\gamma} R^{-2}\left(\psi_{2}(R) \psi_{1}^{\prime}(R)-\psi_{1}(R) \psi_{2}^{\prime}(R)\right) .
\end{aligned}
$$


Assumption (5.18) implies that

$$
b_{1}>0
$$

Now we are going to show that the root $\lambda$ of (5.20) satisfies (5.19) provided the coefficients satisfy (5.21)-(5.24). It is clear that the roots $\lambda$ of (5.20) are given by

$$
\lambda_{ \pm}=\frac{1}{2 a}\left\{-\left(b_{1}+i b_{2}\right) \pm\left(b^{2}-4 a c\right)^{\frac{1}{2}}\right\} .
$$

Let

$$
X=b_{1}^{2}-b_{2}^{2}-4 a c \quad \text { and } \quad Y=b_{1} b_{2} .
$$

Then

$$
b^{2}-4 a c=X+2 i Y .
$$

Moreover, if $x$ and $y$ are real numbers such that

$$
(x+i y)^{2}=X+i Y,
$$

then

$$
x^{2}=\frac{1}{2}\left\{X+\left(X^{2}+4 Y^{2}\right)^{\frac{1}{2}}\right\} .
$$

To show (5.19), it suffices to prove that $b_{1}>|x|$, i.e.,

$$
b_{1}^{2}>x^{2} .
$$

By (5.21) and (5.22), we have

$$
2 b_{1}^{2}-X=b_{1}^{2}+b_{2}^{2}+4 a c>0 .
$$

It is easy to check that

$$
\left(2 b_{1}^{2}-X\right)^{2}-\left(X^{2}+4 Y^{2}\right)=16 a c .
$$

Hence (5.26) follows from (5.21), (5.22), (5.27), and (5.28). The proof is complete.

Next, we prove the following instability results.

Lemma 5.5. Let u be a neutrally stable BC-type stationary solution of (EC) or (EPC) when $\nu=0$. For any $\nu>0$, if $\lambda$ is real and $\lambda>0$, we have

$$
\psi(R, \lambda)>0 \quad \text { and } \quad \psi^{\prime}(R, \lambda)>0 .
$$

Furthermore, we have

$$
\lim _{\lambda \rightarrow 0^{+}} \frac{\psi^{\prime}(R, \lambda)}{\psi(R, \lambda)}=+\infty
$$

and

$$
\lim _{\lambda \rightarrow \infty} \frac{\psi^{\prime}(R, \lambda)}{\psi(R, \lambda)}=+\infty .
$$


Proof. If $\lambda>0$, then $p(r)+\lambda_{\gamma}^{\nu}>0$ in $[1, R]$. Integrating (5.3) from 1 to $r$ and using (5.5) and (5.6), we obtain

$$
r^{-2}\left(p(r)+\lambda \frac{\nu}{\gamma}\right) \psi^{\prime}(r)=\left(p(1)+\lambda \frac{\nu}{\gamma}\right)+\frac{1}{\gamma} \int_{1}^{r}\left\{4 s^{-3} p^{\prime}(s)+\lambda^{2} s^{-2}\right\} \psi(s, \lambda) d s .
$$

If $\lambda^{2}$ is large enough that

$$
\lambda^{2}+4 s^{-1} p^{\prime}(s) \geq 0 \quad \text { in }[1, R],
$$

then (5.5), (5.6), and (5.32) imply

$$
\psi(r, \lambda)>0 \quad \text { and } \quad \psi^{\prime}(r, \lambda)>0 \quad \text { in }[1, R] .
$$

In particular, (5.29) holds.

Now by applying Theorem 5.4, we claim that (5.29) also holds for any $\lambda>$ 0 . Otherwise, by the continuous dependence of $\psi(R, \lambda)$ with respect to $\lambda$, we have $\psi^{\prime}\left(R, \lambda_{1}\right)=0$ or $\psi\left(R, \lambda_{1}\right)=0$ for some $\lambda_{1}>0$. Since (5.18) is satisfied by this $\lambda_{1}$, Theorem 5.4 implies $\lambda_{1}<0$, a contradiction. Hence (5.29) holds for any $\lambda>0$.

To show (5.30), we note that $u$ is neutrally stable and by Proposition A.1 in Appendix A, we have

$$
\lim _{r \rightarrow R^{-}}(R-r)^{q} \psi(r, 0)=c_{0}>0
$$

and

$$
\lim _{r \rightarrow R^{-}}(R-r)^{q+1} \psi^{\prime}(r, 0)=c_{1}>0 .
$$

From (5.35), (5.36), and (5.29), it is not difficult to prove that (5.30) holds. The details of the proof are omitted.

Finally, it remains to prove (5.31). If $\lambda>0$ and is large enough, then (5.3), (5.33), and (5.34) imply that

$$
\psi^{\prime \prime}(r, \lambda)>0 \quad \text { in }[1, R] .
$$

Moreover, by (5.32), there is a positive constant $c_{2}$ that is independent on $\lambda$ such that for a large $\lambda$, we have

$$
\psi^{\prime}(R, \lambda) \geq \lambda c_{2} \int_{r}^{R} \psi(s, \lambda) d s
$$

for $r \in\left[{ }_{2}^{1} R, R\right]$. Now for any $s \in\left[{ }_{2}^{1} R, R\right]$, write

$$
\psi(s, \lambda)=\psi(R, \lambda)+\psi^{\prime}(R, \lambda)(s-R)+{ }_{2}^{1} \psi^{\prime \prime}(\tilde{r}, \lambda)(s-R)^{2}
$$

for some $\tilde{r} \in(s, R)$. Subsituting (5.39) into (5.38) and using (5.37), we obtain

$$
\psi^{\prime}(R, \lambda)\left\{1+\frac{1}{2} \lambda c_{2}(R-r)^{2}\right\} \geq \lambda c_{2} \psi(R, \lambda)(R-r) .
$$


If we choose $r$ such that $(R-r) \lambda^{\frac{1}{2}}=1$, then (5.40) implies that for a large $\lambda$, we have

$$
\psi^{\prime}(R, \lambda) \geq c_{3} \lambda^{\frac{1}{2}} \psi(R, \lambda)
$$

where the positive constant $c_{3}$ is independent of $\lambda$. Hence (5.31) follows. The proof is complete.

For any real $\lambda \notin\left[-\lambda^{*}, 0\right], \psi(r, \lambda)$ is a real function, i.e., $\psi_{2}=\operatorname{Im} \psi \equiv 0 . \kappa_{2}(\lambda)$ is then undetermined for all real $\lambda \notin\left[-\lambda^{*}, 0\right]$. However, we can define $\kappa_{2}(\lambda)$ for these $\lambda$ by going through the following limiting process.

Let $\lambda_{1}$ and $\lambda_{2}$ be real numbers such that $\lambda_{1} \notin\left[-\lambda^{*}, 0\right]$ and $\left|\lambda_{2}\right| \neq 0$ and is sufficiently small. We then have

$$
\psi\left(r, \lambda_{1}+i \lambda_{2}\right)=\psi\left(r, \lambda_{1}\right)+i \lambda_{2} \frac{\partial \psi}{\partial \lambda}\left(r, \lambda_{1}\right)+o\left(\left|\lambda_{2}\right|^{2}\right)
$$

as $\lambda_{2} \rightarrow 0$. Therefore, for any real $\lambda_{1} \notin\left[-\lambda^{*}, 0\right]$, we can define

$$
\kappa_{2}\left(\lambda_{1}\right)=\frac{\partial^{2} \psi}{\partial r \partial \lambda}\left(R, \lambda_{1}\right) / \frac{\partial \psi}{\partial \lambda}\left(R, \lambda_{1}\right) .
$$

It is not difficult to prove that $\kappa_{2}(\lambda)$ is well defined and is continuous for $\lambda \in \mathbf{C}-$ $\left[-\lambda^{*}, 0\right]$.

Now by applying Lemma 5.5, we have that following instability result.

THEOREM 5.6. Let $u$ be a neutrally stable BC-type stationary solution of (EC) or $(\mathrm{EPC})$ when $\nu=0$. Then for any $\nu>0$, there is a positive constant $\kappa^{*}=\kappa^{*}(\nu, u)$ such that for any $\kappa_{1}<-\kappa^{*}$, there is a nonempty open set $U\left(\kappa_{1}, \nu, u\right)$ such that $u$ is unstable with respect to (5.5) and (5.15) for $\left(\kappa_{1}, \kappa_{2}\right)$ with $\kappa_{2} \in U\left(\kappa_{1}, \nu, u\right)$.

Proof. For any $\nu>0$, let

$$
\kappa^{*}(\nu, u)=\min \left\{\frac{\psi^{\prime}(R, \lambda, \nu)}{\psi(R, \lambda, \nu)}: \lambda \in(0, \infty)\right\} .
$$

By (5.30) and (5.31), we have $\kappa^{*}(\nu, u)>0$. If $\kappa_{1}<-\kappa^{*}$, then there is $\lambda_{1}>0$ such that

$$
\frac{\psi^{\prime}\left(R, \lambda_{1}\right)}{\psi\left(R, \lambda_{1}\right)}=-\kappa_{1} .
$$

Let

$$
U\left(\kappa_{1}, \nu, u\right)=\left\{\kappa_{2} \in(-\infty, \infty]:\left(\kappa_{1}, \kappa_{2}\right) \in K_{u}\right\} .
$$

Then (5.42) implies that

$$
\kappa_{2}\left(\lambda_{1}\right) \in U\left(\kappa_{1}, \nu, u\right) .
$$

Thus $U\left(\kappa_{1}, \nu, u\right)$ is nonempty. It is clear that $U\left(\kappa_{1}, \nu, u\right)$ is open, and the result follows. The proof is complete.

Proof of Theorem 1.2. Theorem 1.2 follows from Theorems 5.4 and 5.6.

We now come to (EP). In this case, (5.3) has a singularity at $r=0$ even for $\lambda \notin\left[-\lambda^{*}, 0\right)$. Therefore, we need to modify our argument to obtain a result as in Lemma 5.1. Indeed, the initial conditions (5.5) and (5.6) will be replaced with

$$
r^{-2} \psi(r, \lambda)=0 \quad \text { at } r=0
$$


and

$$
\left(r^{-2} \psi(r, \lambda)\right)^{\prime}=1 \quad \text { at } r=0 .
$$

We then have the following result.

Lemma 5.7. Let $u$ be a B-type solution of (EP). Then the solution $\psi(r, \lambda)$ of (5.3), (5.43), and (5.44) exists in a neighborhood of $r=0$ if $\lambda \neq-\lambda^{*} \equiv{ }_{\nu}^{\gamma} p(0)$. Furthermore, $\psi(\cdot, \lambda)$ has the same property as in Lemma 5.1 .

Proof. Following the same argument as in the proof of Lemma 5.1, we have equation (5.9) for $\tilde{\psi}_{1}$ and $\tilde{\psi}_{2}$ in $r>0$. For $\lambda \neq-\lambda^{*}$, after a careful computation, (5.9) can be written as

$$
\tilde{\psi}_{1}^{\prime \prime}=\left(-\frac{2}{r}+g_{1}\right) \tilde{\psi}_{1}^{\prime}+\left(\frac{2}{r^{2}}+f_{1}\right) \tilde{\psi}_{1}+g_{2} \tilde{\psi}_{2}^{\prime}+f_{2} \tilde{\psi}_{2}
$$

and

$$
\tilde{\psi}_{2}^{\prime \prime}=\left(-\frac{2}{r}+g_{1}\right) \tilde{\psi}_{2}^{\prime}+\left(\frac{2}{r^{2}}+f_{1}\right) \tilde{\psi}_{2}-g_{2} \tilde{\psi}_{1}^{\prime}-f_{2} \tilde{\psi}_{1}
$$

where $g_{j}(r)$ and $r f_{j}(r)$ are continuous (in fact, $C^{2}$ ) at $r=0$ for $j=1,2$. Now $r=0$ is a regular singular point in (5.45) and (5.46). By a standard argument, we can prove that there is bounded solution $\left\{\tilde{\psi}_{1}, \tilde{\psi}_{2}\right\}$ of $(5.45)$ and (5.46). Moreover, they satisfy

$$
\begin{aligned}
& \tilde{\psi}_{1}(r)=a_{0} r+o(r), \\
& \tilde{\psi}_{2}(r)=b_{0} r+o(r)
\end{aligned}
$$

as $r \rightarrow 0^{+}$. The details of the proof are omitted. Now the initial conditions (5.43) and (5.44) imply that $a_{0}$ and $b_{0}$ satisfy

$$
a_{0}=p(0)+\lambda_{1} \frac{\nu}{\gamma} \quad \text { and } \quad b_{0}=\lambda_{2} \frac{\nu}{\gamma} .
$$

Subsituting (5.47) into (5.9), we obtain

$$
\begin{aligned}
& \psi_{1}(r)=r^{3}+o\left(r^{3}\right), \\
& \psi_{2}(r)=O\left(r^{3}\right)
\end{aligned}
$$

as $r \rightarrow 0^{+}$.

The other properties of $\psi(\cdot, \lambda)$ can also be obtained as in proving Lemma 5.1; the details are omitted. The proof is complete.

By arguing as in Theorems 5.4 and 5.6, we can obtain the following stability result for problem (EP).

THEOREM 5.8. Let $u$ be a neutrally stable B-type stationary solution of (EP) when $\nu=0$. Then for any $\nu>0, u$ is stable with respect to (5.43) and (5.15) if $\kappa_{1} \geq 0$ and $\kappa_{2} \geq 0$. On the other hand, there is a positive constant $\kappa^{*}=\kappa^{*}(\nu, u)$ such that for any $\kappa_{1}<-\kappa^{*}$, there is a nonempty open set $U\left(\kappa_{1}, \nu, u\right)$ such that $u$ is unstable with respect to (5.43) and (5.15) for $\left(\kappa_{1}, \kappa_{2}\right)$ with $\kappa_{2} \in U\left(\kappa_{1}, \nu, u\right)$.

Proof. The proof is the same as was used for Theorems 5.4 and 5.6. Therefore, the details are omitted.

Remark 5.9. In their recent work on (EC), Makino et al. [15, 16, 19] showed that when $\gamma>{ }_{3}^{4}$ and $\nu>0, u_{R, \mu}$ is nonlinearly asymptotically stable with respect to small perturbations. Their result is consistent with ours. 
Appendix A. Asymptotic behavior at $\boldsymbol{R}$. In this section, we shall study the asymptotic behavior of a real solution $\psi$ at $R$ for (3.3) with real $\ell$. Let

$$
\tau=R-r \quad \text { and } \quad \tilde{\psi}(\tau)=\psi(r) .
$$

Then $\tilde{\psi}$ satisfies

$$
\tilde{\psi}^{\prime \prime}+\left\{(1+q) \tau^{-1}+g(\tau)\right\} \tilde{\psi}^{\prime}+\tau^{-1} f(\tau) \tilde{\psi}=0 .
$$

For simplicity, we omit the $\sim$ 's and write the last equation as

$$
\psi^{\prime \prime}+\left\{(1+q) \tau^{-1}+g(\tau)\right\} \psi^{\prime}+\tau^{-1} f(\tau) \psi=0, \quad \tau>0,
$$

where $g$ and $f$ are continuous at $\tau=0$.

Then we have the following result concerning the behavior of $\psi$ at 0 .

Proposition A.1. For any $q>1$, let $\psi$ be a solution of (A.1). Then either $\psi$ is bounded at 0 or

$$
\psi(\tau)=\tau^{-q} \hat{\psi}(\tau)
$$

with $\hat{\psi}$ continuous at 0 and $\hat{\psi}(0) \neq 0$. Furthermore, in the former case, $\psi$ is $C^{2}$ at 0 , and in the latter case, we have

$$
\psi^{\prime}(\tau)=-q \tau^{-q-1} \hat{\psi}(0)+o\left(\tau^{-q-1}\right)
$$

as $\tau \rightarrow 0^{+}$.

Proof. If $g$ and $f$ are analytic in a neighborhood of $\tau=0$, then the result is well known; see, e.g., [2]. For completeness, we provide a proof here that assumes only that $g$ and $f$ are continuous at $\tau=0$. Since the proof is elementary, some details are omitted.

For $\tau>0$, let

$$
\psi(\tau)=\tau^{-q} \omega(\tau)
$$

$\omega$ then satisfies

$$
\omega^{\prime \prime}+\left\{(1-q) \tau^{-1}+g\right\} \omega^{\prime}+(f-q g) \tau^{-1} \omega=0, \quad \tau>0 .
$$

Let $G(0)=0$ and $G^{\prime}(\tau)=g(\tau),($ A.5) can then be written as

$$
\left(\tau^{1-q} e^{G} \omega^{\prime}\right)^{\prime}+\tau^{-q} e^{G}(f-q g) \omega=0 .
$$

Fix $\tau_{1}>0$ and let $\tau_{0} \in\left(0, \tau_{1}\right)$ be chosen later. After integrating (A.6) from $\tau$ to $\tau_{0}$, we have

$$
\omega^{\prime}(\tau)=\tau^{q-1} E(\tau) C_{0}+\tau^{q-1} E(\tau) \int_{\tau}^{\tau_{0}} F(s) s^{-q} \omega(s) d s
$$

where

$$
\begin{gathered}
E(\tau)=\exp (-G(\tau)), \quad F(\tau)=(f-q g) \exp (G(\tau)), \\
\text { and } \quad C_{0}=\tau_{0}^{1-q} \omega^{\prime}\left(\tau_{1}\right) \exp \left(G\left(\tau_{1}\right)\right) .
\end{gathered}
$$


We first claim that $\omega$ and $\omega^{\prime}$ are bounded on $(0, \tau]$.

Indeed, let

$$
C_{1}=\left|C_{0}\right| \max _{\tau \in\left[0, \tau_{1}\right]}|E(\tau)| \quad \text { and } \quad C_{2}=\max _{\tau \in\left[0, \tau_{1}\right]}|E(\tau)| \cdot \max _{\tau \in\left[0, \tau_{1}\right]}|F(\tau)| .
$$

Then from (A.7), we have

$$
\left|\omega^{\prime}(\tau)\right| \leq C_{1} \tau^{q-1}+C_{2} \tau^{q-1} \int_{\tau}^{\tau_{0}} s^{-q}|\omega(s)| d s,
$$

which implies that

$$
\left|\omega^{\prime}(\tau)\right| \leq C_{1} \tau^{q-1}+C_{3} \max _{s \in\left[\tau, \tau_{0}\right]}|\omega(s)|
$$

where

$$
C_{3}=C_{2} \cdot(q-1)^{-1} .
$$

Now for any $\tau \in\left(0, \tau_{0}\right)$, substituting (A.9) into

$$
\omega(\tau)=\omega\left(\tau_{0}\right)+\int_{\tau_{1}}^{\tau} \omega^{\prime}(s) d s
$$

we obtain

$$
|\omega(\tau)| \leq\left|\omega\left(\tau_{0}\right)\right|+C_{4}+C_{3} \tau_{0} \max _{s \in\left[\tau, \tau_{0}\right]}|\omega(s)|
$$

where

$$
C_{4}=C_{1} \tau_{1}^{q} .
$$

Now if we choose $C_{3} \tau_{0}<1$, (A.10) then implies

$$
|\omega(\tau)| \leq\left(1-C_{3} \tau_{0}\right)^{-1}\left\{\left|\omega\left(\tau_{0}\right)\right|+C_{4}\right\} .
$$

Hence $\omega$ is bounded on $\left[0, \tau_{1}\right]$. By (A.9), $\omega^{\prime}$ is bounded on $\left[0, \tau_{1}\right]$, which also implies that $\omega$ is continuous at 0 . Now if $\omega(0) \neq 0$, then (A.4) and (A.8) imply (A.3). If $\omega(0)=0$, we shall claim that

$$
|\omega(\tau)| \leq C_{5} \tau^{q}
$$

for some $C_{5}>0$. Indeed, $\omega(0)=0$ and $\omega^{\prime}$ bounded on $\left[0, \tau_{1}\right]$ implies that

$$
|\omega(\tau)| \leq C_{6} \tau
$$

Now substituting (A.12) into (A.9), we have

$$
\left|\omega^{\prime}(\tau)\right| \leq C_{7} \tau^{q-1}+C_{8} \tau
$$

for some $C_{7}>0$ and $C_{8}>0$. Substituting (A.13) into

$$
\omega(\tau)=\int_{0}^{\tau} \omega^{\prime}(s) d s,
$$

we obtain a better estimate for $\omega$ than (A.12). After repeating the processes a finite number of times, (A.15) follows. The proof is complete. 
Appendix B. Friedrichs' criteria. In this section, we recall a useful criterion of Friedrichs [4] for studying the spectra of second-order differential operators that are self-adjoint and singular at their endpoints.

Let $J=(x-, x+) \subset \mathbf{R}^{1}$ be a bounded or unbounded open interval.

Let $a(x), a^{\prime}(x), b(x)$, and $c(x)$ be continuous functions on $J$. Furthermore, $a(x)$ and $c(x)$ are positive on $J$. The eigenvalue equation

$$
-\left(a(x) \phi^{\prime}(x)\right)^{\prime}+b(x) \phi(x)=\lambda c(x) \phi(x)
$$

can be written as

$$
L \phi=\lambda \phi
$$

where

$$
L=c^{-1}(x)\left\{-\frac{d}{d x}\left(a(x) \frac{d}{d x}\right)+b(x)\right\}
$$

Define

$$
h=\left|\int \frac{d x}{a(x)}+C\right|>0
$$

in the neighborhood of $x-$ or $x+$. The constants $C=C_{-}$or $C=C_{+}$should be chosen such that at the endpoint, $h$ is either zero or infinite.

$$
\text { If } h(x-)=0 \quad(\text { or } h(x+)=0),
$$

then we require that

$$
\phi(x-)=0 \quad(\text { or } \phi(x+)=0) .
$$

Otherwise, we need not put conditions on $\phi$ at $x-$ or $x+$.

Define $X=\left\{\phi: J \rightarrow \mathbf{R}^{1}: \phi\right.$ is absolutely continuous and satisfies $\int_{x-}^{x+} c(x) \phi^{2}(x) d x$ $<\infty, \int_{x-}^{x+} a(x) \phi^{\prime 2}(x) d x<\infty$, and $\int_{x-}^{x+}|b(x)| \phi^{2}(x) d x<\infty$ and also satisfies (B.1) if (B.2) holds\} and

$$
(\phi, \psi)=\int_{x-}^{x+} c(x) \phi(x) \psi(x) d x .
$$

Definition B.1. The spectrum of $L$ is called discrete below $\lambda_{*}$ if for every $\lambda^{\prime}<\lambda_{*}$ there exists at most a finite number of mutually orthogonal eigenfunctions $\phi_{\lambda}(x)$ associated with eigenvalue $\lambda \leq \lambda^{\prime}$ such that for every $\phi \in X$ such that

$$
\left(\phi, \phi_{\lambda}\right)=0
$$

we have

$$
(\phi, L \phi) \geq \lambda_{*}(\phi, \phi) .
$$

$L$ is called totally discrete if $L$ possesses a pure point spectrum.

Remark B.2. If the spectrum is discrete below every $\lambda_{*}$, then it is totally discrete.

Define

$$
Z(x)=\frac{1}{c(x)}\left\{b(x)+\frac{1}{4 a(x) h^{2}(x)}\right\}
$$

Friedrichs' criterion can then be stated as follows.

Proposition B.3. 
(I) $L$ is totally discrete if

$$
Z(x) \rightarrow \infty \quad \text { as } x \rightarrow x-\text { and } x \rightarrow x+.
$$

(II) $L$ is discrete below $\lambda_{*}$ if

$$
\liminf Z(x) \geq \lambda_{*} \quad \text { as } x \rightarrow x-\text { and } x \rightarrow x+.
$$

(III) $L$ is not discrete below $\lambda^{*}$ if $Z(x)$ is bounded below and

$$
\lim \sup Z(x)<\lambda^{*}
$$

as either $x \rightarrow x-$ or $x \rightarrow x+$.

(IV) The spectrum of $L$ is discrete below $\lambda_{*}$, unbounded below, if

$$
\begin{aligned}
& \liminf _{x \rightarrow x-} Z(x) \geq \lambda_{*}, \\
& \lim _{x \rightarrow x+} Z(x)=-\infty,
\end{aligned}
$$

and

$$
\Omega=\int_{x_{0}}^{x+}\left\{\begin{array}{c}
c(x) \\
-a(x) Z(x)
\end{array}\right\}^{\frac{1}{2}} d x<\infty
$$

where $x_{0}<x+$ such that $Z(x)<0$ in $\left(x_{0}, x+\right)$. A similar result holds if the roles of $x-$ and $x+$ are interchanged.

Acknowledgments. The author wishes to thank Professors T. P. Liu and T. Makino for several useful discussions about this work.

\section{REFERENCES}

[1] S. Chandrasekhar, An Introduction to the Study of Stellar Structures, University of Chicago Press, Chicago, 1939.

[2] E. A. Coddington and N. Levison, Theory of Ordinary Differential Equations, McGraw-Hill, New York, 1955.

[3] R. Courant and D. Hilbert, Methods of Mathematical Physics, Vols. I and II, Interscience, New York, 1953 and 1962.

[4] K. O. Friedrichs, Criteria for discrete spectra, Comm. Pure Appl. Math., 3 (1950), pp. 439449.

[5] W. C. KUAN AND S. S. Lin, Numbers of equilibria for the equation of self-gravitating isentropic gas surrounding a solid ball, Japan J. Indust. Appl. Math., 13 (1996), pp. 311-331.

[6] T. Makino, On a local existence theorem for the evolution equation of gaseous stars, in Patterns and Waves: Qualitative Analysis of Nonlinear Differential Equations, T. Nishida, M. Mimura, and K. Fujii, eds., North-Holland, Amsterdam, 1986, pp. 459-479.

[7] T. Makino, Blowing up solutions of the Euler-Poisson equation for the evolution of gaseous stars, Transport Theory Statist. Phys., 21 (1992), pp. 615-624.

[8] T. Makino, Mathematical aspects of the Euler-Poisson equation for the evolution of gaseous stars, lecture notes, National Chiao-Tung University, Hsin-chu, Taiwan, 1993.

[9] T. Makino, K. Mizohata, And S. Ukai, The global weak solutions of compressible Euler equation with spherical symmetry, Japan J. Indust. Appl. Math., 9 (1992), pp. 431-449.

[10] T. Makino, K. Mizohata, And S. UKaI, The global weak solutions of compressible Euler equation with spherical symmetry II, Japan J. Indust. Appl. Math., 11 (1994), pp. 417426.

[11] T. Makino and B. Perthame, Sur les solutions à symétric sphérique de l'equation d'EulerPoisson pour l'evolution d'etoiles gazeuses, Japan J. Appl. Math., 7 (1990), pp. 165-170. 
[12] T. Makino And S. TAKeno, Initial boundary value problem for the spherically symmetric motion of isentropic gas, Japan J. Indust. Appl. Math., 11 (1994), pp. 173-183.

[13] T. Makino And S. Ukai, Sur l'existence des solutions locales de l'equation d'Euler-Poisson pour l'evolution gazeuses, J. Math. Kyoto Univ., 27 (1987), pp. 387-399.

[14] T. Makino, S. UkaI, And S. KaWashima, Sur la solution à support compact de l'equation d'Euler compressible, Japan J. Appl. Math., 3 (1986), pp. 249-257.

[15] Š. Matušú-NeČasová, M. OKada, AND T. Makino, Free boundary problem for the equation of spherically symmetric motion of viscous gas II, Japan J. Indust. Appl. Math., 12 (1995).

[16] Š. MatušÙ-NeČasová, M. Okada, And T. Makino, Free boundary problem for the equation of spherically symmetric motion of viscous gas III, preprint.

[17] W.-M. Ni AND R. Nussbaum, Uniqueness and non-uniqueness for positive radial solution of $\Delta u+f(u, r)=0$, Comm. Pure Appl. Math., 38 (1985), pp. 67-108.

[18] W.-M. Ni AND J. SERRIN, Existence and nonexistence theorems for ground states of quasilinear partial differential equations: The anomalous case, Acad. Naz. Lincei, 77 (1986), pp. 231257.

[19] M. OKADA AND T. MaKino, Free boundary problem for the equation of spherically symmetric motion of viscous gas, Japan J. Indust. Appl. Math., 10 (1993), pp. 219-235.

[20] A. D. Rendall, The initial value problem for self-gravitating fluid bodies, in Mathematical Physics X, K. Schmudgen, ed., Springer-Verlag, Berlin, 1992, pp. 471-474. 